\title{
Global Stability Analysis of the Role of Antiretroviral Therapy (ART) Abuse in HIVIAIDS Treatment Dynamics
}

\author{
Bassey Echeng Bassey*, Adagba Odey Henry \\ Department of Industrial Mathematics and Applied Statistics, Ebonyi State University, Abakaliki, Nigeria \\ Email address: \\ awaserex@ymail.com (B. E. Bassey), helena2012@gmail.com (A. O. Henry) \\ ${ }^{*}$ Corresponding author
}

To cite this article:

Bassey Echeng Bassey, Adagba Odey Henry. Global Stability Analysis of the Role of Antiretroviral Therapy (ART) Abuse in HIV/AIDS

Treatment Dynamics. Pure and Applied Mathematics Journal. Vol. 10, No. 1, 2021, pp. 9-31. doi: 10.11648/j.pamj.20211001.12

Received: December 24, 2020; Accepted: February 20, 2021; Published: March 4, 2021

\begin{abstract}
Devastatingly, in spite of the long standing research works on HIV/AIDS infection and treatment dynamics, reviews of existing models clearly shown that the behavioral attitude to treatment consistency by those screened to become aware and those receiving treatment have not been given the desired attention. Moreso, the inconsistency following avoidable treatment truncation and later resumption of treatment by these classes of infectives, which could lead to colossal drug abuse is also not accorded the much expected consideration. Therefore, in this present study, we sought and formulated a nonlinear 6Dimensional deterministic mathematical HIV/AIDS dynamic model that accounted for the global stability analysis of the role of antiretroviral therapy abuse for the treatment of HIV/AIDS epidemic. The model is structured upon dynamical interactions between 6-subpopulations and HI-virus under bilinear control functions with constant screening of the susceptible. It is assumed that the rate of resumption of ART upon truncation is less than initial ART truncation following the incorporation of HIV aware infectives not ready to receive ART treatment and HIV aware infectives with truncated treatment protocol The system mathematical well-posedness was investigated and model reproduction number determined for both off- treatment (with value 0.343 ) and for onset-treatment (with value 0.271 ). We considered the model for off-treatment and thereafter by incorporating LaSalle's invariant principle into classical Lyapunov function method, we presented an approach for the global stability analysis of the role of ART abuse in HIV/AIDS treatment. Furthermore, the analysis and results of this paper presented a dynamic methodological application of bilinear control functions and an impeccable understanding of the fundamental mechanism in HIV/AIDS treatment in the presence of ART abuse. Using in-built Runge-Kutta of order of precision 4 in a Mathcad surface, numerical validity of model is conducted to investigate the study theoretical and analytical predictions. Results shows that application of onset-treatment functions with trend of ART abuse yield tremendous reduction in HIV/AIDS infection epidemic following the recovery rate of the susceptible population with value increasing from 0.5 cells $/ \mathrm{mm}^{3}$ to 1.203 cells $/ \mathrm{mm}^{3}$ within the first 3 months and attained stability of 0.62 cells $/ \mathrm{mm}^{3}$ through the time interval of 2030 months.
\end{abstract}

Keywords: Antiretroviral-Therapy-abuse, Global-Stability-Conditions, Lyapunov-Function, Bilinear-Control-Functions, Upper-Triangular-Matrix, Mass-Action, Theoretical Predictions, Analytical Results

\section{Introduction}

Following the enormity of the present research, we thought it should be worthy to subdivide the introductory aspect into two distinct parts in order to give an insight to the disease historical background and associated literature on mathematical models of HIV/AIDS infection dynamics.

\subsection{Historical Background on HIV Infection}

The human immunodeficiency virus (HIV) is known to belong to the class of viruses called Retroviruses characterized by ribonucleic acid (RNA) as their genetic material. HIV is understood to be the causative agent of the acquired immunodeficiency syndrome (AIDS). The dimension of HIV infection and its treatment dynamics is known to have moved beyond the virus and risk factors 
associated with its transmission to a more detailed understanding of the mechanism associated with the spread distribution and impact of any intervention on the population.

In reality, the magnitude of the untold hardship resulting from HIV infection in the update is firmly relatively unknown, though matching an estimated annual incidence rate of about 24.9 million in 2000 to 37.9 million as at end of June, 2019 (UNAIDS, 2019), [1]. Perturbingly, since the discovery of AIDS in 1981, the epidemic has become a threat to the health and survival of millions of individuals, families and communities worldwide. Until now, despite the effort made by world bodies like the World Health Organization (WHO), United Nation International Children Emergency Fund (UNICEF), United Nation Development Program (UNDP), Joint United Nations Programme on AIDS (UNAIDS) and other recent organizations on HIV in collaboration with several scientific research bodies, there have been no outright medical cure for the deadly disease. Rather available, are a number of preventive and intervention treatment measures in the range of condom use and antiretroviral drugs such as Azidothymidine (AZT), Nucleoside analogues, Non-nucleoside analogues, Reverse transcriptase inhibitors (RTI), Protease inhibitors (PIs) and Fusion inhibitors (FIs), [2]. These drugs are administered as preventive and suppressive measures to victims in order to keep the virus inactive for some time. Notably, these therapeutic treatment have appeared promising in retarding the spread and replication of HIV and HIV related infection, noting that the aspect of prevention remain the most effective strategy against the spread of HIV/AIDS epidemic, [3].

The most vulnerable at risk of acquiring the deadly disease are individual from developing countries of Asia and Africa having records of sexual contacts with HIV infected people, homosexual, bisexual men, intravenous drug abuser user/sharing of household and medical accessories and persons transfused with contaminated blood, [4]. Devastating is the fact that the spread of HIV infection is seen to be much earlier and easier in these parts of the continents when compared with the rest of the world. A situation that can be attributed to lack effective utilization of available drugs, arising from low knowledgeable and abject poverty. Moreso, the unassuming consequences arising from complete lack of outright cure, incessant drug abuse and the negative status as the incurable disease, forms the integral factor of the present investigation. HIV is basically transmitted through two major routes: horizontal routes consisting of sexual intercourse (both homosexual and heterosexual) contacts, blood transfusion, intravenous drug users and contaminated injecting equipment; and vertical transmission route (mother-to-child). The latter route involves the transmission of the virus from mother-tochild via the utero (during pregnancy), during labor and delivery as well as through breastfeeding, [5-7].

\subsection{Literature Review on HIV Mathematical Models}

The problem of human immunodeficiency virus (HIV) remains an important component of mankind and the solutions are often based on the role of mathematical modeling in understanding of the mechanism of the spread of the virus among the population. For instance, taking from early investigation, mathematical model had been used to conduct preliminary study of the transmission dynamics of HIV as the causative agent of AIDS as well as the discussion of transmission model for AIDS, [8, 9]. The effort of these researchers were further strengthened following the extensively studied of the transmission dynamics of HIV infection, [10]. Since then, several mathematical models on HIV transmission and treatment methodologies have been developed. Using ordinary differential equation, HIV/AIDS treatment with the incorporation of sexual behavioral change, condom use and avoidance of high-risk sexual practices as indicators have been investigated, [11]. Result here showed that lowering sexual behavior lowers' prevalence and incidence rate. Moreso, the ability of condom to stop AIDS epidemic was studied, [12. Latin hypercube sampling and partial rank correlation coefficient was adopted with result showing that preventability has negative effect on the epidemic.

It is known that mathematical modeling have been used to study the contributive role of condom use and antiretroviral therapy (ART) and the results analyzed using known and modern numerical methods. For instance, a two-sex mathematical model was formulated to investigate the prevention of HIV/AIDS in a varying population, [13]. This study was further extended following the investigation of the impact of non-compliance in condom use as preventive measure among heterosexual population, [14]. Attributing knowledge, attitude, practice and determinant as indicators, random sampling technique was explored to study the use of condom for the control of HIV/AIDS in Northern West Ethiopia, [15]. The results indicated that $78.9 \%$ of infected patients exhibited knowledgeable and consistent condom use for the prevention of the spread of HIV/AIDS alongside unwanted pregnancies. Other related mathematical models for the use of condom only, as preventive measure include those of models $[6,16,17]$. In using multiple preventive and suppressive measures, other several mathematical models comes into focus. For example, condom use and antiretroviral therapy incorporating male and female in the use of condom have been studied, [18]. Moreso, mathematical investigation for the application of counseling and ART for the transmission dynamics of HIV infection was conducted, [19]. In the model [20], the author resorted to explore condom use and counseling for the eradication of the spread of the deadly disease.

As a motivating factors to the present investigation, recent innovative findings have been considered. For instance, using mathematical model, the effect of screening of unaware HIV/AIDS infectives on the spread of HIV infection without any control function (treatments) have been investigated, [21]. Improving on the above finding while sustaining the screening method, similar model with the incorporation of condom use by the susceptible and application of control function (ART) by aware infective class was proposed and studied, [22]. Notably, a review of these models shows that the behavioral attitude to treatment consistency by those who were screened 
to become aware and receiving treatment was not considered. Moreso, the inconsistency following avoidable treatment truncation and possible resumption of treatment by these classes of infectives, which could lead to colossal drug abuse was not accorded the desired attention. Therefore, this present study motivated by the aforementioned limitations, seek to formulate as an extended version, a 6-dimensional deterministic compartmental HIV/AIDS mathematical dynamic model with the incorporation of aware infective class not ready to receive treatment, aware infective on consistent ART treatment and those that are inconsistent with ART (leading to ART abuse), while sustaining the screening method and treatment control functions. That is, using mathematical model, the present study is anticipated to enhance new pathways and to proffer compactible treatment schedule for the fight against the deadly disease. Moreso, the study is intended to provide insight to the global stability analysis and to evaluate its impact with a view to possibly overturn the adverse role of ART abuse, using non-linear differential mathematical dynamic model.

Resourcefully, the entire study is partitioned into 7 sections with section 1 devoted to the introductory aspect. Section 2 consider quantitatively, the material and methods adopted for the study, which routinely include: the basic mathematical problem statement and formulation of proposed model. In section 3, we investigate the mathematical wellposedness characterized by system invariant region and system positivity of solutions. The existence of model equilibria and global stabilities conditions is treated in section 4 . In section 5 , we conducted numerical validity of our theoretical predictions and analytical results. Obtained results are then discussed and analyzed in section 6 and finally, section 7 is devoted to succinct observed conclusion and remarks. Objectively, the present study propose to project an insight to the behavioral attitude of aware infectives under consistent and inconsistent treatment control functions using stability analysis.

\section{Material and Methods}

In this section, we quantitatively consider the material and methods adopted for the study. This routinely include: the basic mathematical problem statement and the formulation of proposed model.

\subsection{Problem Statement and Mathematical Formulation of Model Equations}

The present study bring to bear, two closely related models as a lead-way for the smooth formulation of our present model. Concisely, the effect of screening of unaware infectives on the spread of HIV infection was investigated, [20]. The model considered four stages of the population in HIV/AIDS progression following the absence of treatment and post-exposure intervention. These sub-populations include: the susceptible or HIV negative population $S(t)$, HIV positives or infectives that do not know they are infected
$I_{1}(t)$, HIV positives that know they are infected $I_{2}(t)$ and the full-blown AIDS population $A(t)$. The epidemiological derived equations of the model is given as:

$$
\begin{aligned}
\frac{d S}{d t} & =Q_{0}-\left(\frac{\beta_{1} S I_{1}+\beta_{2} S I_{2}}{N}\right)-d S \\
\frac{d I_{1}}{d t} & =\frac{\beta_{1} S I_{1}}{N}+\frac{\beta_{2} S I_{2}}{N}-(\theta+\delta+d) I_{1} \\
\frac{d I_{2}}{d t} & =\theta I_{1}-(\delta+d) I_{2} \\
\frac{d A}{d t} & =\delta I_{1}+\delta I_{2}-(\alpha+d) A
\end{aligned}
$$

with $S(0)=S_{0}>0, I_{1}(0)=I_{1(0)} \geq 0, I_{2}(0)=I_{2(0)} \geq 0, A(0)=A_{0} \geq 0$ and having population under consideration defined by $N=S+I_{1}+I_{2}+A$. For further details of the model descriptions and assumption, we refer readers to the cited authors.

Presumably, taking into account of the fact that the above model was developed in the absence of treatment and postexposure intervention but rather with the effect of screening of unaware infectives, similar model with the introduction of treatment functions both at the susceptible stage (condom use) and those who were screen and placed under ART treatment was proposed and studied, [22]. For clarity of presentation, while urging readers to access the model details and assumptions, the differential equations for the model was derived as:

$$
\left\{\begin{array}{l}
\frac{d S}{d t}=\varphi_{0}-\beta_{i} S-\mu S, \\
\frac{d I_{1}}{d t}=\beta_{i} S-(\mu+\delta+\theta) I_{1}, \\
\frac{d I_{2}}{d t}=\theta I_{1}-(\mu+\delta+\pi) I_{2}, \\
\frac{d A}{d t}=\delta\left(I_{1}+I_{2}\right)+\pi I_{2}-(\mu+\alpha) A
\end{array},\right.
$$

where,

$$
\beta_{i}=\left(1-u_{1}\right)\left(\frac{\beta_{1} c_{1} I_{1}+\beta_{2} c_{2} I_{2}+\beta_{3} c_{3} A}{N(t)}\right) .
$$

Equation (3) is called the model force of infection otherwise known as the system mass action.

A clear analysis of the above two proposed motivating models paves the way for our proposed investigation.

From epidemiological point of view, equation (2) used stability theory of differential equations and had dwelled on the effect of screening of the unaware HIV/AIDS infectives on the spread of HIV infection. Here, no direct incorporation of treatment function, noting that upon been screened and becoming aware, sexual interaction were bound to change resulting to reduction of the endemicity of the infection. On the other hand, improving on the above findings led to a study of similar model with the incorporation of condom use by the susceptible and application of treatment control function (ART) by the aware infectives class, while sustaining the screening 
method, [22]. The model was analyzed using the theory of Volterra-Lyapunov stable matrices on classical method of Lyapunov functions. The result lead to the fundamental understanding of the mechanism in HIV/AIDS infection.

None-the-less, review of these models indicated the obvious of the fact that the behavioral attitude to treatment consistency by those who had been screened to become aware and receiving treatment was not considered. Moreso, the inconsistency by avoidable truncation of treatment measures and possible resumption of treatment by these classes of infectives, which could lead to colossal drug abuse was not accorded the desired attention by these studies. Thus, on the account of overcoming these scientific limitations from the aforementioned two motivating models, the present study is necessitated.

\subsection{Formulation of Proposed (Extended) Model}

Captivated by the innovative findings of the above two motivating models and its associated limitations, the present study seeks to present an extended version of these two models. That is, we formulate a deterministic compartmental model that comprehensively account for the transmission dynamics of HIV/AIDS infection with the incorporation of aware infectives class but not ready to receive treatment, those who are consistent in ART treatment and those who are inconsistent with ART treatment, leading to abuse of the therapy (ART). Thus, the present study in addition to the existing assumptions, proposes the following assumptions, which completely enhance the present extended model.

Assumption 1

i. The state variable $I_{2}$ is redefined to be HIV positive individuals who are aware of their infection but are not ready to take the ART.

ii. ART abuse individuals are those receiving the therapy but stop at a time and then resume later.

iii. The contact rate of susceptible with full-blown AIDS is much less than ART abuse infectives which in turn is much less than those ART none abusers; and subsequently much less than aware infectives and which is much less than the unaware HIV infectives (i.e., $\beta_{5}<\beta_{4}<\beta_{3}<\beta_{2}<\beta_{1}$ ).

iv. Clearance rate due to infection only occur at full-blown up AIDS, i.e., $\alpha>0$ for AIDS population at time $t$.

v. Resumption rate for use of ART is less than rate at which those that use ART and then stop the therapy (abuse) and in turn less than those receiving ART (i.e., $\left.a_{3}<a_{2}<a_{1}\right)$.

Of note, following the introduction of a new concept with incorporated state variables, the population under investigation is subdivided into the following 6 compartments: susceptible individuals who are not HIV positive but may be infected if exposed $S(t)$, HIV positive (infective) individuals who are unaware of their HIV status at time $t I_{1}(t)$, number of HIV positive individuals who are aware of their infection but not ready to receive ART $I_{2}(t)$, number of HIV positive (infective) individuals receiving ART $T(t)$, number of HIV positive (infective) individuals under ART who have stop receiving the therapy but later resume treatment $T_{A}(t)$ and full-blown AIDS population at time $t A(t)$. The epidemiological differential equations for the system basic model is derive as:

$$
\left\{\begin{array}{l}
\frac{d S}{d t}=\varphi_{0}-\beta_{i} S-\mu S, \\
\frac{d I_{1}}{d t}=\beta_{i} S-(\mu+\delta+\theta) I_{1}, \\
\frac{d I_{2}}{d t}=\theta I_{1}-\left(\mu+\delta+a_{1}\right) I_{2}, \\
\frac{d T}{d t}=a_{1} I_{2}+a_{3} T_{A}-\left(\mu+\pi+a_{2}\right) T, \\
\frac{d T_{A}}{d t}=a_{2} T-\left(\mu+\delta+a_{3}\right) T_{A}, \\
\frac{d A}{d t}=\delta\left(I_{1}+I_{2}+T_{A}\right)+\pi T-(\mu+\alpha) A,
\end{array}\right.
$$

where

$$
\beta_{i(i=1, \ldots, 5)}=\left(1-u_{1}\right)\left(\frac{\beta_{1} c_{1} I_{1}+\beta_{2} c_{2} I_{2}+\beta_{3} c_{3} T+\beta_{4} c_{4} T_{A}+\beta_{5} c_{5} A}{N(t)}\right),
$$

with $N(t)=S+I_{1}+I_{2}+T+T_{A}+A$.

It can then be said that with equation (5) depicting the system mass action (force of infection), equation (4) is an inert epidemiological equation representing the mathematical expression for the transmission dynamics of HIV/AIDS population under ART abuse model. Schematically, system (4) is depicted by Figure 1 below:

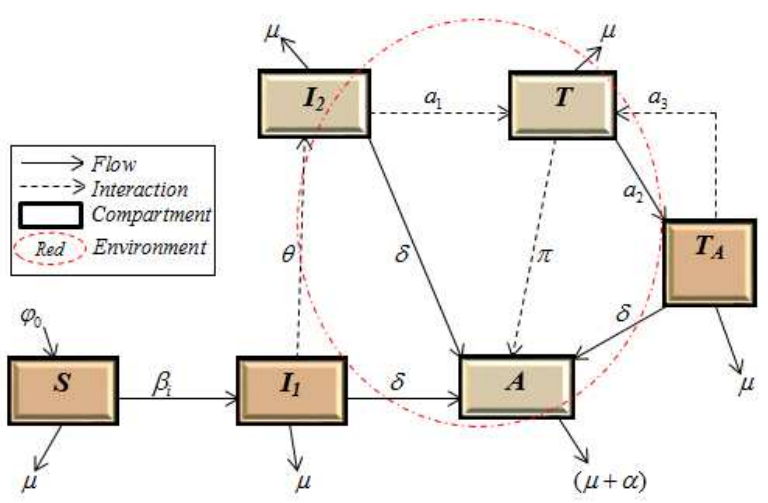

Figure 1. Schematic diagram of HIV/AIDS transmission dynamics with ART abuse (misuse).

The parameters associated with equation 4 (as used in figure 1) are defined as follows: $\varphi_{0}$ - recruitment rate (population source), $\beta_{i=1, \ldots, 5}$ - probability of interaction by susceptible with various infectives, $c_{i=1, \ldots, 5}-$ sexual contact by susceptible with various infectives, $\lambda$ - successful condom use, $\theta$ - rate at which the unaware becomes aware, $\pi$ progression rate of aware infective to AIDS, $\delta$ - rate at which infectives develops AIDS. Others include: $\alpha$ - AIDS induce 
death rate, $\mu$ - natural death rate, $a_{1}$ - rate of ART receive by $T(t), a_{2}$ - rate of abuse of ART by $T_{A}(t)$ and $a_{3}$ - resumption rate of ART by $T_{A}(t)$.

Since the present model is a complete representation of a set of living organism, then we must show that the model is mathematically well-posed. That is, we establish in the next section, the mathematical properties and model analysis for our derived equations.

\section{Mathematical Properties and Analysis of Model}

To show that the characteristic properties of the model equation is mathematically well-posed, is to determine the invariant region representing the entire system as nonnegative and the existence of positivity of the system solutions. We initiate this investigation by showing that the differential sum of the equations of model (4) is a function of natural birth and death rates as well as the clearance rate due to infection at full-blown AIDS.

Thus, from equation (4) the differential sum is given by

$$
\begin{gathered}
\frac{d N}{d t}=\frac{d S}{d t}+\frac{d I_{1}}{d t}+\frac{d I_{2}}{d t}+\frac{d T}{d t}+\frac{d T_{A}}{d t}+\frac{d A}{d t} \\
=\varphi_{0}-\beta_{i} S-\mu S+\beta_{i} S-(\mu+\delta+\theta) I_{1}+\theta I_{1}-\left(\mu+\delta+a_{1}\right) I_{2} \\
+a_{1} I_{2}+a_{3} T_{A}-\left(\mu+\pi+a_{2}\right) T+a_{2} T-\left(\mu+\delta+a_{3}\right) T_{A} \\
+\delta\left(I_{1}+I_{2}+T_{A}\right)+\pi T-(\mu+\alpha) A
\end{gathered}
$$

Simplifying, we obtain

$$
\begin{gathered}
\frac{d N}{d t}=\varphi_{0}-\mu S-\mu I_{1}-\mu I_{2}-\mu T-\mu T_{A}-\mu A-\alpha A, \\
=\varphi_{0}-\mu\left(S+I_{1}+I_{2}+T+T_{A}+A\right)-\alpha A \\
\frac{d N}{d t}=\varphi_{0}-\mu N-\alpha A,
\end{gathered}
$$

since, $N(t)=S+I_{1}+I_{2}+T+T_{A}+A$.

Equation (6) is an index with which we can then show that the invariant region representing the entire system are all positive.

\subsection{System Invariant Region}

Remarkably, it can be said that all the state variables and parameters are non-negative for $t>0$, since the system (4) represent a set of human population. Invariably, this region can be investigated using the result of the following theorem.
Theorem$$
1
$$
The
closed
set

$\mathfrak{R}_{D}=\left\{\left(S, I_{1}, I_{2}, T, T_{A}, A\right) \in \mathbb{R}_{+}^{6}: N \leq \frac{\varphi_{0}}{\mu}\right\} \quad$ is positively invariant and attracting with respect to the system (4).

Proof
Here, we invoke the classical approach adopted in existing proves of invariant region [23]. Then, if we consider equation (6), we have

$$
\frac{d N}{d t}=\varphi_{0}-\mu N-\alpha A
$$

In the absence of mortality due to HIV/AIDS infection, where the population is completely free from HIV/AIDS infection i.e., $\alpha=0$, we obtain

$$
\frac{d N}{d t} \leq \varphi_{0}-\mu N
$$

It follows that

$$
\frac{d N}{d t}+\mu N \leq \varphi_{0}
$$

This is a first order homogenous differential inequality. We solve the above equation by finding the integrating factor

$$
\text { (IF) i.e., } e^{\mu \int d t}=e^{\mu t} \text {. }
$$

Then, multiplying through by the integrating factor, we have

$$
e^{\mu t} \frac{d N}{d t}+e^{\mu t} \mu N \leq e^{\mu t} \varphi_{0}
$$

or

$$
\frac{d}{d t}\left[e^{\mu t} N\right] \leq e^{\mu t} \varphi_{0}
$$

Integrating both sides yields

$$
e^{\mu t} N \leq e^{\mu t} \frac{\varphi_{0}}{\mu}+C
$$

where $C$ is a constant of integration. Simplifying gives

$$
N(t) \leq \frac{\varphi_{0}}{\mu}+C e^{-\mu t}
$$

Now, applying initial condition when $t=0$, i.e., $N(t)=N(0)$, we have

$$
N(0) \leq \frac{\varphi_{0}}{\mu}+C
$$

or

$$
N(0)-\frac{\varphi_{0}}{\mu} \leq C
$$

Substituting for $C$, we obtain

$$
N(t) \leq \frac{\varphi_{0}}{\mu}+\left[N(0)-\frac{\varphi_{0}}{\mu}\right] e^{-\mu t},
$$


where $N(0)$ is the initial population at time $t=t_{0}=0$. This gives $N(t) \leq N(0)$ as $t \rightarrow 0$ and $N(t) \leq \frac{\varphi_{0}}{\mu}$ as $t \rightarrow \infty$.

Applying the Birkhof and Rota's theorem on differential inequality when $t \rightarrow \infty$, we arrive at $0 \leq N(t) \leq \frac{\varphi_{0}}{\mu}$ for all $t \geq 0$, [24]. But from the system (4), we have,

$$
\frac{d S}{d t}+\frac{d I_{1}}{d t}+\frac{d I_{2}}{d t}+\frac{d T}{d t}+\frac{d T_{A}}{d t}+\frac{d A}{d t}=0
$$

which imply that

$$
\frac{d N}{d t}=0
$$

Integrating on both sides yields

$$
N=C \text {. }
$$

Since,

$$
N=S+I_{1}+I_{2}+T+T_{A}+A=1 .
$$

It follows that $C=1$, implying that the population is constant, positive and equal to 1 . Hence, all the feasible solutions of the system (4) enter the region

$$
\mathfrak{R}_{D}=\left\{\left(S, I_{1}, I_{2}, T, T_{A}, A\right) \in \mathfrak{R}_{+}^{6}: S+I_{1}+I_{2}+T+T_{A}+A=1\right\} .
$$

Therefore, the region is positive and attracting, implying that the model is both mathematically well-posed in the region $\Re_{D}$ and epidemiologically meaningful.

\subsection{Positivity of Solutions}

The following theorem give the required verification for the positivity of solutions of the system variables.

Theorem 2 Assume the initial conditions $\left\{S(0), I_{1}(0), I_{2}(0), T(0), T_{A}(0), A(0)\right\} \in \mathfrak{R}_{+}^{6}$. Then the solution set $\left\{S(t), I_{1}(t), I_{2}(t), T(t), T_{A}(t), A(t)\right\}$ of the model (4) remain positive for all $t \geq 0$.

Proof

We invoke existing results for positivity of solutions [25, 26]. Then, from the first equation of system (4), we have

$$
\frac{d S}{d t}=\varphi_{0}-\beta_{i} S-\mu S=\varphi_{0}-\left(\beta_{i}+\mu\right) S
$$

Taking the differential, we have

$$
\frac{d S}{d t} \leq \varphi_{0}-\left(\beta_{i}+\mu\right) S
$$

It follows that

$$
\frac{d S}{d t}+\left(\beta_{i}+\mu\right) S \leq \varphi_{0}
$$

Again, this is a first order homogeneous differential inequality.
Solution of the equation is obtained by applying the integrating factor $I F=e^{\left(\beta_{i}+\mu\right) \int d t}=e^{\left(\beta_{i}+\mu\right) t}$. Therefore, the positivity of solution for the susceptible population is derived as:

$$
S(t) \leq \frac{\varphi_{0}}{\left(\beta_{i}+\mu\right)}+\left[S(0)-\frac{\varphi_{0}}{\left(\beta_{i}+\mu\right)}\right] e^{-\left(\beta_{i}+\mu\right) t} .
$$

or

$$
S(t) \leq S(0) e^{-\left(\beta_{i}+\mu\right) t}+\frac{\varphi_{0}}{\left(\beta_{i}+\mu\right)}\left[1-e^{-\left(\beta_{i}+\mu\right) t}\right]
$$

Then, we see that since $S(t)>0$ as $t=0$ and $S(t)>0$ as $t \rightarrow \infty$, implying that $S(t)>0, \forall t \geq 0$.

Similarly, from the second to the sixth equations of model (4), the positivity of solutions for the state variables are derived as follows: For unaware infectives $I_{1}(t)$, we have,

$$
I_{1}(t) \geq I_{1}(0) e^{-(\mu+\delta+\theta) t} .
$$

We observed that since $(\mu+\delta+\theta)>0$ then $I_{1} \geq 0$ as $t=0$, and $I_{1}(t) \geq 0$ as $t \rightarrow \infty$. Therefore, $I_{1}(t) \geq 0, \forall t \geq 0$.

The positivity of solution for the aware infectives but not ready to receive is derived as

$$
I_{2}(t) \geq I_{2}(0) e^{-\left(\mu+\delta+a_{1}\right) t} .
$$

Obviously, since $\left(\mu+\delta+a_{1}\right)>0$ then $I_{2} \geq 0$ as $t=0$ and $I_{2}(t) \geq 0$ as $t \rightarrow \infty$. Therefore, $I_{2}(t) \geq 0, \forall t \geq 0$. Furthermore, for HIV positive (infectives) individuals receiving ART, the positivity of solution is derived as:

$$
T(t) \geq T(0) e^{-\left(\mu+\pi+a_{2}\right) t},
$$

where $\left(\mu+\pi+a_{2}\right)>0$. Then $T \geq 0$ if $t=0$ and $T(t) \geq 0$ as $t \rightarrow \infty$. Therefore, $T(t) \geq 0, \forall t \geq 0$.

For the positivity of solution for HIV infectives that abuse the use of therapy (ART), we have

$$
T_{A}(t) \geq T_{A}(0) e^{-\left(\mu+\delta+a_{3}\right) t},
$$

which represents the aware infective who had initiated treatment but discontinue this treatment with probability of resuming such treatment later. Since $\left(\mu+\delta+a_{3}\right)>0$, then $T_{A} \geq 0$ provided $t=0$ and $T_{A}(t) \geq 0$ as $t \rightarrow \infty$. Therefore, $T(t) \geq 0, \forall t \geq 0$. Finally, the positivity of solution for the infectious virus (full-blown AIDS) is derived as

$$
A(t) \geq A(0) e^{-(\mu+\alpha) t},
$$

which implies that $A(t) \geq 0, \forall t \geq 0$. Hence, we conclude that $S(t)>0, I_{1}(t) \geq 0, I_{2}(t) \geq 0, T(t) \geq 0, \quad T_{A}(t) \geq 0, A(t) \geq 0$ $\forall t \geq 0$, implying that all the state variable are all positive. This completes the proof.

Next, we investigate the stability analysis of our extended basic model, which is revolve around the existence of equilibrium points of the system and computation of the 
system reproduction number. This is obvious as it serves as a tool for the determination of HIV/AIDS transmission dynamics under ART abuse.

\section{Stability Analysis of the Extended Model}

In this section, we investigate the existence of model equilibria and further study quantitatively, their stabilities conditions.

\subsection{Existence of Equilibrium States}

For the existence of an equilibrium state, it is assumed that model (4) is at its steady state i.e.,

$$
\frac{d S}{d t}+\frac{d I_{1}}{d t}+\frac{d I_{2}}{d t}+\frac{d T}{d t}+\frac{d T_{A}}{d t}+\frac{d A}{d t}=0
$$

This implies that we equate the right-hand side derivatives to zero as presented hereof:

$$
\left\{\begin{array}{l}
0=\varphi_{0}-\left(\beta_{i}+\mu\right) S, \\
0=\beta_{i} S-m_{1} I_{1}, \\
0=\theta I_{1}-m_{2} I_{2}, \\
0=a_{1} I_{2}+a_{3} T_{A}-m_{3} T, \\
0=a_{2} T-m_{4} T_{A}, \\
0=\delta\left(I_{1}+I_{2}+T_{A}\right)+\pi T-m_{5} A
\end{array} .\right.
$$

Equivocally, from equation (8), disease-free equilibrium (DFE) for system (4) exists if $u_{1}=0$ with other controls held constant. Then, in computing the DFE, we let $H^{0}$ denote the DFE such that each of the equations of model (4) is equated to zero i.e. at disease-free equilibrium, no infection and no recovery. The implication is that

$$
I_{1}^{0}=0, I_{2}^{0}=0, T^{0}=0, T_{A}^{0}=0, A^{0}=0
$$

and

$$
H^{0}=\left(S^{0}, I_{1}^{0}, I_{2}^{0}, T^{0}, T_{A}^{0}, A^{0}\right)=0 .
$$

Thus, using equations (8) and (9) to solve for $H^{0}$ yields

$$
0=\varphi_{0}-\left(\beta_{i}+\mu\right) S,
$$

i.e.,

$$
\left(\beta_{i}+\mu\right) S=\varphi_{0}
$$

This imply that

$$
S^{0}=\frac{\varphi_{0}}{\left(\beta_{i}^{*}+\mu\right)} .
$$

Also, from the second equation, we have,

$$
0=\beta_{i} S-m_{1} I_{1}
$$

which implies that

$$
m_{1} I_{1}=\beta_{i} S
$$

i.e.,

$$
I_{1}^{0}=\frac{\beta_{i}^{*} S^{0}}{m_{1}}
$$

Substituting the value of $S^{0}$ into the above, we obtain

$$
I_{1}^{0}=\frac{\beta_{i}^{*}\left(\frac{\varphi_{0}}{\beta_{i}^{*}+\mu}\right)}{m_{1}} .
$$

Simplifying, we have,

$$
I_{1}^{0}=\left(\frac{\varphi_{0}}{m_{1}}\right) \frac{\beta_{i}^{*}}{\beta_{i}^{*}+\mu} .
$$

Similarly, from the third equation of (8), we have,

$$
0=\theta I_{1}-m_{2} I_{2} \text {. }
$$

This imply that

$$
m_{2} I_{2}=\theta I_{1},
$$

i.e.,

$$
I_{2}^{0}=\frac{\theta I_{1}^{0}}{m_{2}} .
$$

Using the value for $I_{1}^{0}$, we obtain

$$
I_{2}^{0}=\frac{\theta\left\{\left(\frac{\varphi_{0}}{m_{1}}\right) \frac{\beta_{i}^{*}}{\beta_{i}^{*}+\mu}\right\}}{m_{2}} .
$$

Simplifying, we have,

$$
I_{2}^{0}=\left(\frac{\varphi_{0} \theta}{m_{1} m_{2}}\right) \frac{\beta_{i}^{*}}{\beta_{i}^{*}+\mu} .
$$

In similar approach, we solve for $T^{0}, T_{A}^{0}$ and $A^{0}$, which comprehensively yields the corresponding values for $H^{0}$ i.e.,

$$
\left\{\begin{array}{l}
S^{0}=\frac{\varphi_{0}}{\beta_{i}^{*}+\mu} \\
I_{1}^{0}=\left(\frac{\varphi_{0}}{m_{1}}\right) \frac{\beta_{i}^{*}}{\beta_{i}^{*}+\mu} \\
I_{2}^{0}=\left(\frac{\varphi_{0} \theta}{m_{1} m_{2}}\right) \frac{\beta_{i}^{*}}{\beta_{i}^{*}+\mu} \\
T^{0}=\left(\frac{\varphi_{0} \theta m_{4} a_{1}}{\left(m_{3} m_{4}-a_{2} a_{3}\right) m_{1} m_{2}}\right) \frac{\beta_{i}^{*}}{\beta_{i}^{*}+\mu} \\
T_{A}^{0}=\left(\frac{\varphi_{0} \theta a_{1} a_{2}}{\left(m_{3} m_{4}-a_{2} a_{3}\right) m_{1} m_{2}}\right) \frac{\beta_{i}^{*}}{\beta_{i}^{*}+\mu} \\
A^{0}=\left(\frac{\pi \theta m_{4} a_{1}+\delta \theta a_{1} a_{2}+\delta\left(\theta+m_{2}\right)\left(m_{3} m_{4}-a_{2} a_{3}\right)}{\left(m_{3} m_{4}-a_{2} a_{3}\right) m_{1} m_{2} m_{5}}\right) \varphi_{0} \frac{\beta_{i}^{*}}{\beta_{i}^{*}+\mu}
\end{array},\right.
$$


where

$$
\left\{\begin{array}{cc}
m_{1}=\mu+\delta+\theta, & m_{2}=\mu+\delta+a_{1}, \\
m_{3}=\mu+\pi+a_{2}, & m_{4}=\mu+\delta+a_{3}, \\
m_{5}=\mu+\alpha & .
\end{array}\right.
$$

Now, since $\beta_{i}^{*}=0$ define the point at which HIV/AIDS free equilibrium (HAFE) exists, then using equations (8) and (10), equation (11) representing the equilibrium point denoted by $H^{0}$ becomes

$$
H^{0}=\left(S^{0}, I_{1}^{0}, I_{2}^{0}, T^{0}, T_{A}^{0}, A^{0}\right)=\left(\frac{\varphi_{0}}{\mu}, 0,0,0,0,0\right) .
$$

Equation (13) clearly defines the equilibrium point of the system, where no infection exists. Now, with the introduction of virus infection into the system, we are required to determine the pattern of the transmission dynamics. This process demands that we establish the system reproduction number denoted by $R_{0}$.

\subsection{Computation of HIV/AIDS Control Reproduction Number, $R_{0}$}

The basic reproduction number, $R_{0}$ of an infectious system is the expected number of secondary cases produced in a completely susceptible population by a typical HIV/AIDS infectious individual, [27]. It is a threshold quantity that determines the elimination or spread of HIV/AIDS in a population. That is, if $R_{0}<1$, then the implication is that on average, an infected individual can generate less than one HIV/AIDS infective, thus, the disease cannot spread. On the contrary, when $R_{0}>1$, it leads to outbreak of disease epidemic. Therefore, in this present study, we adopt existing approach in finding the HIV/AIDS control reproduction number, $R_{0}$ in an ART abuse scenario, [27]. Thus, by such a method, and taking equation (12) into account, the system (4) is rearranged with the infected components first as presented hereof:

$$
\left\{\begin{array}{l}
\frac{d I_{1}}{d t}=\beta_{i} S-m_{1} I_{1}, \\
\frac{d I_{2}}{d t}=\theta I_{1}-m_{2} I_{2}, \\
\frac{d T}{d t}=a_{1} I_{2}+a_{3} T_{A}-m_{3} T, \\
\frac{d T_{A}}{d t}=a_{2} T-m_{4} T_{A}, \\
\frac{d A}{d t}=\delta\left(I_{1}+I_{2}+T_{A}\right)+\pi T-m_{5} A, \\
\frac{d S}{d t}=\varphi_{0}-\beta_{i} S-\mu S
\end{array}\right\}
$$

From equations (5) and (14), we adopt the Next Generation matrices defined by

$$
\left[\frac{\partial F_{i}}{\partial x_{j}}\left(H^{0}\right)\right] \cdot\left[\frac{\partial V_{i}}{\partial x_{j}}\left(H^{0}\right)\right]^{-1}
$$

where the notations $F_{i}$ and $V_{i}$, denotes the matrices of new infections in compartment $i$ and the transfer terms at HIV/AIDS free equilibrium into compartment $i$ while $H^{0}$ is the HAFE. That is,

$$
F_{i=1, . .5}=\left(\begin{array}{ccccc}
\lambda \beta_{1} c_{1} & \lambda \beta_{2} c_{2} & \lambda \beta_{3} c_{3} & \lambda \beta_{4} c_{4} & \lambda \beta_{5} c_{5} \\
0 & 0 & 0 & 0 & 0 \\
0 & 0 & 0 & 0 & 0 \\
0 & 0 & 0 & 0 & 0 \\
0 & 0 & 0 & 0 & 0
\end{array}\right)
$$

and

$$
V_{i=1, \ldots, 5}=\left(\begin{array}{ccccc}
m_{1} & 0 & 0 & 0 & 0 \\
-\theta & m_{2} & 0 & 0 & 0 \\
0 & -a_{1} & m_{3} & -a_{3} & 0 \\
0 & 0 & -a_{2} & m_{4} & 0 \\
-\delta & -\delta & -\pi & -\delta & m_{5}
\end{array}\right)
$$

with

$$
V^{-1}=\left(\begin{array}{ccccc}
\frac{1}{m_{1}} & 0 & 0 & 0 & 0 \\
\frac{\theta}{m_{1} m_{2}} & \frac{1}{m_{2}} & 0 & 0 & 0 \\
D_{1} & D_{2} & D_{3} & D_{4} & 0 \\
D_{5} & D_{6} & D_{7} & D_{8} & 0 \\
D_{9} & D_{10} & D_{11} & D_{12} & \frac{1}{m_{5}}
\end{array}\right),
$$

where

$$
\begin{aligned}
& D_{1}=\frac{m_{4} \theta a_{1}}{m_{1} m_{2}\left(m_{3} m_{4}-a_{2} a_{3}\right)}, D_{2}=\frac{m_{4} a_{1}}{m_{2}\left(m_{3} m_{4}-a_{2} a_{3}\right)}, D_{3}=\frac{m_{4}}{m_{3} m_{4}-a_{2} a_{3}}, \\
& D_{4}=\frac{a_{3}}{m_{3} m_{4}-a_{2} a_{3}}, D_{5}=\frac{a_{1} a_{2} \theta}{m_{1} m_{2}\left(m_{3} m_{4}-a_{2} a_{3}\right)}, D_{6}=\frac{a_{1} a_{2}}{m_{2}\left(m_{3} m_{4}-a_{2} a_{3}\right)}, \\
& D_{7}=\frac{a_{2}}{m_{3} m_{4}-a_{2} a_{3}}, D_{8}=\frac{m_{3}}{m_{3} m_{4}-a_{2} a_{3}}, D_{9}=\frac{\pi \theta m_{4}+\delta \theta a_{1} a_{2}+\left(m_{3} m_{4}-a_{2} a_{3}\right)\left(\theta+m_{2}\right) \delta}{m_{1} m_{2} m_{5}\left(m_{3} m_{4}-a_{2} a_{3}\right)}, \\
& D_{10}=\frac{\pi a_{1} m_{4}+\delta a_{1} a_{2}+\delta\left(m_{3} m_{4}-a_{2} a_{3}\right)}{m_{2} m_{5}\left(m_{3} m_{4}-a_{2} a_{3}\right)}, D_{11}=\frac{\pi m_{4}+\delta a_{2}}{m_{5}\left(m_{3} m_{4}-a_{2} a_{3}\right)} \\
& \text { and } D_{12}=\frac{\pi a_{3}+\delta a_{1} a_{2}+\delta m_{3}}{m_{5}\left(m_{3} m_{4}-a_{2} a_{3}\right)}
\end{aligned}
$$

define the indicated matrix entries as seen in the vector $V^{-1}$ and $m_{i(i=1, \ldots, 5)}$ represented by equation (12).

Thus, the HIV/AIDS control reproduction number $R_{0}$ with ART abuse as establish by the next generation matrix $F V^{-1}$ is 
derived and computed as:

$$
R_{0}=\rho\left(F V^{-1}\right)=\frac{\lambda \beta_{1} c_{1}}{m_{1}}+\frac{\lambda \beta_{2} c_{2} \theta}{m_{1} m_{2}}+\lambda \beta_{3} c_{3} D_{1}+\lambda \beta_{4} c_{4} D_{5}+\lambda \beta_{5} c_{5} D_{9} .
$$

Or

$$
R_{0}=\sum_{j=1}^{5}\left(\lambda R_{j}\right)
$$

where $j=1, \ldots, 5$ represents the reproduction numbers for $I_{1}, I_{2}, T, T_{A}$ and $A$ respectively. This is to say that $R_{1}=\frac{\lambda \beta_{1} c_{1}}{m_{1}}, R_{2}=\frac{\lambda \beta_{2} c_{2} \theta}{m_{1} m_{2}}, R_{3}=\lambda \beta_{3} c_{3} D_{1}, R_{4}=\lambda \beta_{4} c_{4} D_{5} \quad$ and $R_{5}=\lambda \beta_{5} c_{5} D_{9}$ with $\lambda=1-u_{1}$.

At this point, it will be more explicit if we could define our reproduction number and its implications in terms of offtreatment and during onset-treatment scenarios. Let $R_{0(1)}$ denote the reproduction number for off-treatment scenario, such that all control functions are all zeros i.e. $u_{1}=0, a_{1}=0, a_{3}=0$. Then, equation (15) can be redefine as:

$$
R_{0(1)}=\sum_{j=1}^{2}\left(R_{j}\right)
$$

or

$$
R_{0(1)}=\rho\left(F V^{-1}\right)=\frac{\beta_{1} c_{1}}{m_{1}}+\frac{\beta_{2} c_{2} \theta}{m_{1} m_{2}},
$$

since, $\lambda=1, D_{1}=0, D_{5}=0$ and $D_{9}=0$. By computation, $R_{0(1)}=0.343$, i.e. $R_{0(1)}<1$, implying that the HAFE is locally asymptotically stable (LAS - relatively high). On the other hand, if we let $R_{0(2)}$ denotes the system reproduction number for onset-treatment scenario such that $u_{1}>0, a_{1}>0, a_{3}>0$, then equation (15) holds and can be redefine as:

$$
R_{0(2)}=\sum_{j=1}^{5}\left(\lambda R_{j}\right)
$$

or

$$
R_{0(2)}=\rho\left(F V^{-1}\right)=\frac{\lambda \beta_{1} c_{1}}{m_{1}}+\frac{\lambda \beta_{2} c_{2} \theta}{m_{1} m_{2}}+\lambda \beta_{3} c_{3} D_{1}+\lambda \beta_{4} c_{4} D_{5}+\lambda \beta_{5} c_{5} D_{9},
$$

where, $\quad \lambda=\left(1-u_{1}\right), D_{1}>0, D_{5}>0, D_{9}>0 \quad$ and $\quad u_{1}>0$. Computing equation (17) shows that $R_{0(2)}=0.271$, i.e., $R_{0(2)}<1$, indicates that the HAFE is locally asymptotically stable (LAS - relatively low).

From the above, it is clear that the HAFE for the transmission dynamics of HIV/AIDS model (4) with ART abuse in the presence of onset-treatment is locally asymptotically stable since $R_{0}<1$ and will be unstable if
$R_{0}>1$. From equation (15), it is obvious that we can access the contributions of $I_{1}, I_{2}, T, T_{A}$ and $A$ in terms of $\beta_{1}, \beta_{2}, \beta_{3}, \beta_{4}$ and $\beta_{5}$ respectively. Of note, equation (15) defines $R_{j},(j=1, \ldots, 5)$ as the respective reproduction numbers of each of the infectives with corresponding values computed as follows:

$I_{1} \simeq R_{1}=\frac{\lambda \beta_{1} c_{1}}{m_{1}}=0.092, I_{2} \simeq R_{2}=\frac{\lambda \beta_{2} c_{2} \theta}{m_{1} m_{2}}=0.053$,

$T \simeq R_{3}=\lambda \beta_{3} c_{3} D_{1}=5.763 \times 10^{-4}$,

$T_{A} \simeq R_{4}=\lambda \beta_{4} c_{4} D_{5}=1.107 \times 10^{-3}$ and

$A \simeq R_{5}=\lambda \beta_{5} c_{5} D_{9}=0.124$,

where

$$
R_{0(2)}=R_{1}+R_{2}+R_{3}+R_{4}+R_{5}=0.271<1,
$$

which further explain the varying contributions of the asymptomatic, symptomatic and full-blown AIDS compartments to the overall model reproduction number $R_{0}$.

Then, it is seen from the above equations for $R_{j}$ that $R_{5}>R_{1}>R_{2}>R_{4}>R_{3}$, implying that for large infective population, the full-blown AIDS population contributes more to viral load transmission when compared to the contribution from the unaware infectives $I_{1}$, with declining contributive trend down to those of $T(t)$, which imperatively sustain the disease endemicity in the population with $\beta_{i}>0$. That is HAFE is asymptotically stable (i.e. $R_{0}<1$ ). Moreso, the above evaluation completely agrees with our assumptions, where $\beta_{5}<\beta_{4}<\beta_{3}<\beta_{2}<\beta_{1}$ and as well $a_{3}<a_{1}$. Furthermore, since the class that abuse the use of therapy are much more than the class that eventually resume ART treatment (i.e. $a_{3}<a_{2}$ ), then the contribution from the population of ART abuse $T_{A}(t)$ to the sustenance of HIV endemicity is evidently higher when compared with those of $T(t)$. Compartments of these two infectives from Figure 1, clearly depict the aforementioned analysis.

Now, having explicitly defined $R_{0}$ and its contributive role to HIV/AIDS transmission dynamics with ART abuse, we consider next, the system stability analysis.

\subsection{Local Stability of HIV/AIDS Free Equilibrium (HAFE)}

Here, we digest the local stability of HAFE using the linearization process. Of note, local stability of HAFE is often investigated using the eigenvalues or trace-determinant criteria of linearization process. This approach is known as the Jacobian matrix or simply "The Jacobian." The Jacobian matrix is defined as the matrix of all first-order partial derivative of a vector-valued function, [28]. In more clear terms, equilibrium point of a system is said to be locally asymptotically stable if the Jacobian matrix evaluated at that point has a negative trace and a positive determinant or has negative eigenvalues, [29]. From mathematical point of view, 
local asymptotic stability implies the start of an arbitrary trajectories, which are close to the equilibrium point but never meet the equilibrium point. Hence, the local stability of the HAFE is determined by using the result of the following theorem.

Theorem 3 The HIV/AIDS free equilibrium of the system (4) is locally asymptotically stable when $R_{0}<1$ and unstable for $R_{0}>1$.

Proof

Consider the system model (4) and let

$$
s=\frac{d S}{d t}, i_{1}=\frac{d I_{1}}{d t}, i_{2}=\frac{d I_{2}}{d t}, t=\frac{d T}{d t}, t_{A}=\frac{d T_{A}}{d t} \text { and } \hat{a}=\frac{d A}{d t} .
$$

Then, the model equation (4) becomes

$$
\left\{\begin{array}{l}
s=\varphi_{0}-\left(1-u_{1}\right)\left(\frac{\beta_{1} c_{1} I_{1}+\beta_{2} c_{2} I_{2}+\beta_{3} c_{3} T+\beta_{4} c_{4} T_{A}+\beta_{5} c_{5} A}{N(t)}\right) S-\mu S, \\
i_{1}=\left(1-u_{1}\right)\left(\frac{\beta_{1} c_{1} I_{1}+\beta_{2} c_{2} I_{2}+\beta_{3} c_{3} T+\beta_{4} c_{4} T_{A}+\beta_{5} c_{5} A}{N(t)}\right) S-(\mu+\delta+\theta) I_{1}, \\
i_{2}=\theta I_{1}-\left(\mu+\delta+a_{1}\right) I_{2}, \\
t=a_{1} I_{2}+a_{3} T_{A}-\left(\mu+\pi+a_{2}\right) T, \\
t_{A}=a_{2} T-\left(\mu+\delta+a_{3}\right) T_{A}, \\
\hat{a}=\delta\left(I_{1}+I_{2}+T_{A}\right)+\pi T-(\mu+\alpha) A .
\end{array}\right.
$$

By applying classical linearization process to equation (19), the steady state defined by the Jacobian matrix $J$, is given by

$$
J=\left(\begin{array}{cccccc}
\frac{\partial s}{\partial S} & \frac{\partial s}{\partial I_{1}} & \frac{\partial s}{\partial I_{2}} & \frac{\partial s}{\partial T} & \frac{\partial s}{\partial T_{A}} & \frac{\partial s}{\partial A} \\
\frac{\partial i_{1}}{\partial S} & \frac{\partial i_{1}}{\partial I_{1}} & \cdot & \cdot & \cdot & \cdot \\
\frac{\partial i_{2}}{\partial S} & \cdot & \frac{\partial i_{2}}{\partial I_{2}} & \cdot & \cdot & \cdot \\
\cdot & \cdot & \cdot & \frac{\partial t}{\partial T} & \cdot & \cdot \\
\cdot & \cdot & \cdot & \cdot & \frac{\partial t_{A}}{\partial T_{A}} & \cdot \\
\frac{\partial \hat{a}}{\partial S} & \cdot & \cdot & \cdot & \cdot & \frac{\partial \hat{a}}{\partial A}
\end{array}\right),
$$

which imply from equation (19) that

$$
J=\left(\begin{array}{cccccc}
-\left(\mu+\beta_{i}\right) & -\lambda \beta_{1} c_{1} \frac{S}{N} & -\lambda \beta_{2} c_{2} \frac{S}{N} & -\lambda \beta_{3} c_{3} \frac{S}{N} & -\lambda \beta_{4} c_{4} \frac{S}{N} & -\lambda \beta_{5} c_{5} \frac{S}{N} \\
\beta_{i} & \lambda \beta_{1} c_{1} \frac{S}{N} & \lambda \beta_{2} c_{2} \frac{S}{N} & \lambda \beta_{3} c_{3} \frac{S}{N} & \lambda \beta_{4} c_{4} \frac{S}{N} & \lambda \beta_{5} c_{5} \frac{S}{N} \\
0 & \theta & -m_{2} & 0 & 0 & 0 \\
0 & 0 & a_{1} & -m_{3} & a_{3} & 0 \\
0 & 0 & 0 & a_{2} & -m_{4} & 0 \\
0 & \delta & \delta & \pi & \delta & -m_{5}
\end{array}\right),
$$

with the corresponding upper triangular matrix obtained from the elementary row-operation for HAFE given by

$$
J_{T}^{\circ}=\left(\begin{array}{cccccc}
-\mu & -\lambda \beta_{1} c_{1} & -\lambda \beta_{2} c_{2} & -\lambda \beta_{3} c_{3} & -\lambda \beta_{4} c_{4} & -\lambda \beta_{5} c_{5} \\
0 & -\left(m_{1}-\lambda \beta_{1} c_{1}\right) & \lambda \beta_{2} c_{2} & \lambda \beta_{3} c_{3} & \lambda \beta_{4} c_{4} & \lambda \beta_{5} c_{5} \\
0 & \theta & -W_{1} & \theta \lambda \beta_{3} c_{3} & \theta \lambda \beta_{4} c_{4} & \theta \lambda \beta_{5} c_{5} \\
0 & 0 & 0 & -\xi_{1} & \xi_{2} & a_{1} \theta \lambda \beta_{5} c_{5} \\
0 & 0 & 0 & 0 & -q_{1} & a_{1} a_{2} \theta \lambda \beta_{5} c_{5} \\
0 & 0 & 0 & 0 & 0 & -\zeta
\end{array}\right),
$$

where $W_{1}=m_{2}\left(m_{1}-\lambda \beta_{1} c_{1}\right)-\theta \lambda \beta_{2} c_{2}, \xi_{1}=m_{3}\left[m_{2}\left(m_{1}-\lambda \beta_{1} c_{1}\right)-\theta \lambda \beta_{2} c_{2}\right]-a_{1} \theta \lambda \beta_{3} c_{3}$,

$$
\begin{gathered}
\xi_{2}=m_{2}\left(m_{1}-\lambda \beta_{1} c_{1}\right) a_{3}+a_{1} \theta \lambda \beta_{4} c_{4}=m_{2} m_{1}\left(1-\lambda \beta_{1} c_{1}\right) a_{3}+a_{1} \theta \lambda \beta_{4} c_{4}>0 \\
q_{1}=m_{4}\left[m_{3} m_{2}\left(m_{1}-\lambda \beta_{1} c_{1}\right) a_{3}-a_{1} \theta \lambda \beta_{3} c_{3}\right]-a_{2}\left[m_{2}\left(m_{1}-\lambda \beta_{1} c_{1}\right) a_{3}+a_{1} \theta \lambda \beta_{4} c_{4}\right] \\
\zeta=q_{1} q_{3}-q_{2} a_{1} a_{2} \theta \lambda \beta_{5} c_{5}
\end{gathered}
$$

with

$$
\begin{gathered}
q_{2}=\xi_{1}\left[\delta\left(\lambda \beta_{1} c_{1}+\lambda \beta_{4} c_{4}\right)\left(m_{2}\left(m_{1}-\lambda \beta_{1} c_{1}\right)-\theta \lambda \beta_{2} c_{2}\right)+\delta \theta \lambda \beta_{4} c_{4}\left(\lambda \beta_{1} c_{1}+\lambda \beta_{2} c_{2}\right)\right] \\
+\xi_{2}\left[\left(m_{2}\left(m_{1}-\lambda \beta_{1} c_{1}\right)-\theta \lambda \beta_{2} c_{2}\right)\left(\pi \lambda \beta_{1} c_{1}+\delta \lambda \beta_{3} c_{3}\right)+\delta \theta \lambda \beta_{3} c_{3}\left(\lambda \beta_{1} c_{1}+\lambda \beta_{2} c_{2}\right)\right] \\
\text { and } q_{3}=\xi_{1}\left[W_{1}\left(m_{5}\left(m_{1}-\lambda \beta_{1} c_{1}\right)-\delta \lambda \beta_{5} c_{5}\right)-\delta \lambda \beta_{5} c_{5}\left(\lambda \beta_{1} c_{1}+\lambda \beta_{2} c_{2}\right)\right] \\
-\theta a_{1} a_{2} \lambda \beta_{5} c_{5}\left[W_{1}\left(\pi\left(m_{1}-\lambda \beta_{1} c_{1}\right)+\delta \lambda \beta_{3} c_{3}\right)+\delta \theta \lambda \beta_{3} c_{3}\left(\lambda \beta_{1} c_{1}+\lambda \beta_{2} c_{2}\right)\right]
\end{gathered}
$$

Of note, the above quantities are simply the expansions for the entries (elements) as contain by the Jacobian matrix (21). Form equation (21), we observed from the upper triangular matrix that the major diagonal elements ultimately remain the eigenvalues of the characteristic equation $\operatorname{det}\left(J_{T}^{0}-\lambda I\right)=0$, [24]. Thus,

$$
\begin{gathered}
\lambda_{1}=-\mu, \\
\lambda_{2}=-\left(m_{1}-\lambda \beta_{1} c_{1}\right)=-m_{1}\left(1-R_{1}\right)<0, \text { iff } R_{1}<1, \\
\lambda_{3}=-\left(m_{2}\left(m_{1}-\lambda \beta_{1} c_{1}\right)-\theta \lambda \beta_{2} c_{2}\right)=-m_{2} m_{1}\left(1-R_{1}-R_{2}\right)<0, \\
\text { iff } R_{1}+R_{2}<1, \\
\lambda_{4}=-\xi=-\left(m_{3}\left[m_{2}\left(m_{1}-\lambda \beta_{1} c_{1}\right)-\theta \lambda \beta_{2} c_{2}\right]-a_{1} \theta \lambda \beta_{3} c_{3}\right), \\
\lambda_{4}=-m_{3} m_{2} m_{1}\left(1-R_{1}-R_{2}-R_{3}\left(\frac{m_{3} m_{4}-a_{2} a_{3}}{\left.m_{3} m_{4}\right)}\right)<0,\right. \\
\lambda_{5}=\zeta=-m_{2} m_{1}\left(m_{3} m_{4}-a_{2} a_{3}\right)\left(1-R_{1}-R_{2}-R_{3}-R_{4}\right)<0, \\
\text { provided } \sum_{j=1}^{4} R_{j}<1
\end{gathered}
$$

and

$\lambda_{6}=\zeta=-m_{2} m_{1}\left(m_{3} m_{4}-a_{2} a_{3}\right) q_{3}\left(1-R_{1}-R_{2}-R_{3}-R_{4}-R_{5} \frac{q_{2}}{q_{3}} \phi\right)<0$,

where $\phi=\frac{a_{1} a_{2} m_{5}}{\pi \theta a_{1} m_{4}+\delta\left(\theta a_{1} a_{2}+\left(\theta+m_{2}\right)\left(m_{3} m_{4}-a_{2} a_{3}\right)\right)}$.

Therefore, 
$\lambda_{6}=-m_{1} m_{2}\left(m_{3} m_{4}-a_{2} a_{3}\right) q\left(1-R_{0}\right)<0$, iff $\quad R_{0}<1$,

noting that $\sum_{j=1}^{4} R_{j}+R_{5} \frac{q_{2}}{q_{3}} \phi=R_{0}$.

Therefore, we have shown that the eigenvalues are all negative. Hence, we prove that the HAFE of system (4) is LAS for all $R_{0}<1$.

Next, if infection persists in the population, then endemic equilibrium point is bound to exist. This, we have to verify in the following subsection.

\subsection{Existence and Stability of HIV/AIDS Endemic Equilibrium (Haee) Point}

Obviously, with the presence of the disease in the population i.e. $I_{1} \neq 0, I_{2} \neq 0, T \neq 0, T_{A} \neq 0, A \neq 0$, then the model equation (4) has an equilibrium point known as endemic equilibrium point. It is the existence of this equilibrium point we shall consider hereof.

\subsubsection{Existence of HAEE Point}

Assume $H^{*}=\left(S^{*}, I_{1}^{*}, I_{2}^{*}, T^{*}, T_{A}^{*}, A^{*}\right)$ be the HIV/AIDS endemic equilibrium of the system (4). If we equate equation (6) to zero and using the result of equation (11), we see that the differential sum of the system model (4) at HAEE $\left(H^{*}\right)$ is derived as, [22]

$$
\varphi_{0}-\mu N-\alpha A=0
$$

This implies that at HAEE, the differential sum of population understudy is obtain as

$$
\mu N^{*}=\varphi_{0}-\alpha A^{*} \text {. }
$$

Or

$$
N^{*}=\frac{\varphi_{0}-\alpha A^{*}}{\mu},
$$

which corresponds to the fact that at equilibrium, $\beta_{i}^{*}=0$. If $\beta_{i}^{*}>0$, then there exists disease endemicity and system (4) can be define in terms of disease control reproduction number $R_{0}$ of equation (15) as:

$$
\beta_{i}^{*}\left(1+\beta_{i}^{*} \varphi_{2}-R_{0}\right)=0
$$

from which we obtain

$$
\beta_{i}^{*}=\frac{R_{0}-1}{\varphi_{2}}
$$

The quantity $\varphi_{2}$ in equation (23) is defined using equation (12) as:

$$
\varphi_{2}=\frac{1}{m_{1}}+\frac{\theta}{m_{1} m_{2}}+\frac{m_{4} \theta a_{1}}{m_{1} m_{2}\left(m_{3} m_{4}-a_{2} a_{3}\right)}+\frac{\theta a_{1} a_{2}}{m_{1} m_{2}\left(m_{3} m_{4}-a_{2} a_{3}\right)}+\varphi_{1},
$$

with

$$
\varphi_{1}=\frac{\pi \theta m_{4} a_{1}+\delta \theta a_{1} a_{2}+\delta\left(\theta+m_{2}\right)\left(m_{3} m_{4}-a_{2} a_{3}\right)}{\left(m_{3} m_{4}-a_{2} a_{3}\right) m_{1} m_{2} m_{5}}
$$

noting that $m_{3} m_{4}-a_{2} a_{3}=a_{2}(\mu+\delta)+\left(\mu+\delta+a_{3}\right)$.

Thus, substituting the value of $\beta_{i}^{*}$ in (11), defines the HIV/AIDS endemic equilibrium. Clearly, equation (23) further affirm the definition of the system force of infection in terms of the model reproduction number. Hence, the following result holds.

Theorem 4 The system (4) exhibits a HIV/AIDS endemic equilibrium in the population if and only if $R_{0}>1$.

\subsubsection{Local Stability of $\mathrm{HAEE}$}

Using existing approach as obtain in local stability analysis of HAFE, we transformed the Jacobian matrix (20) with upper triangular matrix given as:

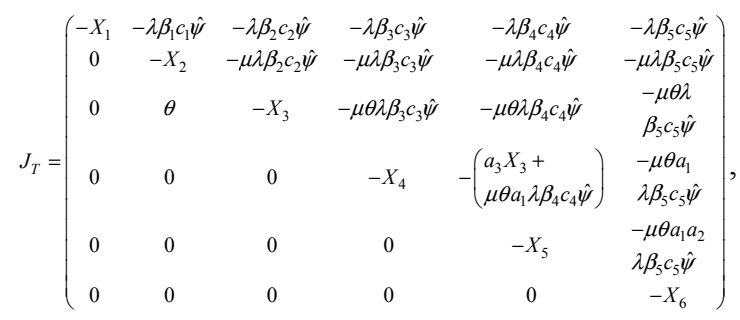

where $\hat{\psi}=\frac{S}{N}$ and $X_{i=1, \ldots .6}$ defined as follows:

$$
\begin{gathered}
X_{1}=\mu+\beta_{m}, X_{2}=\left(\mu+\beta_{m}\right)\left(\lambda \beta_{1} c_{1} \hat{\omega}\right)+\beta_{m} \lambda \beta_{1} c_{1} \hat{\omega}, \\
X_{3}=m_{2} X_{2}-\mu \theta \lambda \beta_{2} c_{2} \hat{\omega}, X_{4}=m_{3} X_{3}-\mu \theta a_{1} \lambda \beta_{3} c_{3} \hat{\omega}, \\
X_{5}=m_{4} X_{4}-a_{2}\left(a_{3} X_{3}+\mu \theta a_{1} \lambda \beta_{4} c_{4} \hat{\omega}\right), \\
X_{6}=-a_{2} a_{1} \mu \theta \lambda \beta_{5} c_{5} \hat{\omega}\left(d_{2} X_{4}+d_{1}\left(a_{3} X_{3}+\mu \theta a_{1} \lambda \beta_{4} c_{4} \hat{\omega}\right)\right)
\end{gathered}
$$

$+\left(m_{4} X_{4}+a_{2}\left(a_{3} X_{3}+\mu \theta a_{1} \lambda \beta_{4} c_{4} \hat{\omega}\right)\right)\left(d_{3} X_{4}-d_{1} \mu \theta a_{1} \lambda \beta_{5} c_{5} \hat{\omega}\right)$,

with

$$
\begin{gathered}
d_{1}=X_{3}\left(\pi X_{2}+\delta \mu \lambda \beta_{3} c_{3} \hat{\omega}\right)+\delta \mu \lambda \beta_{5} c_{5} \hat{\omega}\left(X_{2}+\delta \mu \lambda \beta_{2} c_{2} \hat{\omega}\right) \\
d_{2}=X_{3}\left(X_{2}+\mu \lambda \beta_{4} c_{4} \hat{\omega}\right)+\delta \mu \theta \lambda \beta_{4} c_{4} \hat{\omega}\left(X_{2}+\mu \lambda \beta_{2} c_{2} \hat{\omega}\right)
\end{gathered}
$$

and

$d_{3}=-\delta \mu \theta \lambda \beta_{5} c_{5} \hat{\omega}\left(X_{2}+\mu \lambda \beta_{2} c_{2} \hat{\omega}\right)+X_{3}\left(m_{3} X_{2}-\mu \lambda \beta_{5} c_{5} \hat{\omega}\right)$.

Therefore, we observed from the upper triangular matrix that all the diagonal elements ultimately remain the eigenvalues of the matrix (25) with $\lambda_{i=1, \ldots, 6}$ defined as follows:

$$
\lambda_{1}=-X_{1}=-\left(\mu+\beta_{i}\right)=-\left(\mu+\frac{R_{0}-1}{\varphi_{2}}\right)<0, \text { iff } R_{0}>1,
$$




$$
\begin{aligned}
& \lambda_{2}=-X_{2}=-m_{1}\left\{\mu\left(R_{1} \hat{\omega}-1\right)+\left(\frac{R_{0}-1}{\varphi_{2}}\right)\left(2 R_{1} \hat{\omega}-1\right)\right\}<0, \\
& \text { iff } R_{0}>R_{1}>1 \text {, } \\
& \lambda_{3}=-X_{3}=-m_{1} m_{2}\left\{\begin{array}{l}
\mu\left(\hat{\omega}\left(R_{1}-R_{2}\right)-1\right) \\
+\left(\frac{R_{0}-1}{\varphi_{2}}\right)\left(2 R_{1} \hat{\omega}-1\right)
\end{array}\right\}<0, \\
& \text { iff } R_{0}>R_{1}>R_{2}>1 \text {, } \\
& \lambda_{4}=-X_{4}=-m_{1} m_{2} m_{3}\left\{\begin{array}{l}
\left(\frac{R_{0}-1}{\varphi_{2}}\right)\left(2 R_{1} \hat{\omega}-1\right) \\
+\mu\left[\hat{\omega}\left(R_{1}-R_{2}-R_{3}\left(\frac{m_{3} m_{4}-a_{2} a_{3}}{m_{3} m_{4}}\right)\right)\right]-1
\end{array}\right\}<0, \\
& \text { iff } R_{0}>R_{1}>R_{2}>R_{3}>1 \text {, } \\
& \lambda_{5}=-X_{5}=-m_{1} m_{2}\left(m_{3} m_{4}-a_{2} a_{3}\right)\left\{\begin{array}{l}
\left(\frac{R_{0}-1}{\varphi_{2}}\right)\left(2 R_{1} \hat{\omega}-1\right) \\
+\mu\left[\hat{\omega}\left(R_{1}-R_{2}-R_{3}-R_{4}\right)\right]-1
\end{array}\right\}<0, \\
& \text { iff } R_{0}>R_{1}>R_{2}>R_{3}>R_{4}>1
\end{aligned}
$$

and

$$
\lambda_{6}=-X_{6}<0, \text { iff } \quad R_{6}>0 .
$$

Thus, since all the eigenvalues are negative, we conclude as stated in the theorem below:

Theorem 5 The HIV/AIDS endemic equilibrium of the system (4) is locally asymptotically stable provided that $R_{0}>1$ and unstable otherwise.

\subsection{Global Stability Analysis}

In this sub-section, we identify some basic concepts and notations necessary for the discussion of the system global stability, which include: HIV/AIDS free equilibrium (HAFE) and the global stability of the HIV/AIDS endemic equilibrium (HAEE) under ART abuse for $\alpha=0$

\subsubsection{Identification of Basic Concepts and Notations}

In order to show that system endemic equilibrium is globally asymptotically stable, it becomes necessary to introduce some basic concepts and notations aimed at facilitating our global stability analysis.

Notation 1

We write a matrix $A>0(<0)$, if $A$ is symmetric positive definite (or symmetric negative definite). Then, the following fundamental result on matrix stability holds.

Lemma 1 Let $A$ be a $n \times n$ real matrix. Then all the eigenvalues of $A$ have negative (positive) real parts if and only if there exists a matrix $H>0$ such that $H A+A^{T} H^{T}<0(>0)$.

Lemma 2 Consider a disease model system written in the form:

$$
\left\{\begin{array}{l}
\frac{d X_{1}}{d t}=F\left(X_{1}, X_{2}\right) \\
\frac{d X_{2}}{d t}=G\left(X_{1}, X_{2}\right)
\end{array}\right.
$$

with $G\left(X_{1}, X_{2}\right)=0$, where $X_{1} \in \Re^{m}$ denotes (by its components) the uninfected populations and $X_{2} \in \mathfrak{R}^{n}$ denotes (by its components) the infectious population; $X_{0}=\left(X_{1}^{E}, 0\right)$ denotes the HAEE of the system. Also, assume the following conditions:

$\left(\mathrm{C}_{1}\right)$ For $\frac{d X_{1}}{d t}=F\left(X_{1}, 0\right), X_{1}^{E}$ is globally asymptotically stable,

$\left(\mathrm{C}_{2}\right) \quad G\left(X_{1}, X_{2}\right)=A X_{2}-\hat{G}\left(X_{1}, X_{2}\right)$ with $\hat{G}\left(X_{1}, X_{2}\right) \geq 0$ for $\left(X_{1}, X_{2}\right) \in \Omega$, where the Jacobian matrix $A=\frac{\partial G}{\partial X_{2}}\left(X_{1}^{E}, 0\right)$ has all non-negative off-diagonal elements and $X$ is the region where the model makes biological sense. Then, the HAEE, $X_{0}=\left(X_{1}^{E}, 0\right)$ is globally asymptotically stable provided that $R_{0}<1$.

Lemma 3 Let $D=\left|\begin{array}{ll}d_{11} & d_{12} \\ d_{21} & d_{22}\end{array}\right|$ be a $2 \times 2$ matrix. Then, $D$ is stable iff $d_{11}<0, d_{22}<0$ and $\operatorname{det}(D)=d_{11} d_{22}-d_{12} d_{21}>0$.

The above lemma 3 is explained by the following definition for higher dimension matrix.

Definition 1 A non-singular $n \times n$ matrix $A$ is diagonally stable (or positive stable) if there exists a positive diagonal $n \times n$ matrix $M$ such that $M A+A^{T} M^{T}>0$.

\subsubsection{Global Stability of HAFE}

We prove the system global stability for HAFE by invoking existing theorem and its result, [30].

Theorem 6 The fixed point $H^{0}=\left(\frac{\varphi_{0}}{\mu}, 0,0,0,0,0\right)$ is global asymptotically stable equilibrium of system (4) provided that $R_{0}<1$ and the assumption of equation (26) satisfied.

Proof

Applying lemma 3 to model (4), then we consider

$$
X_{1}=S, X_{2}=\left[\begin{array}{c}
I_{1} \\
I_{2} \\
T \\
T_{A} \\
A
\end{array}\right] \text {. }
$$

When $I_{1}=I_{2}=T=T_{A}=A=0$, the uninfected subsystem (i.e. the equation $S$ ) becomes 


$$
\frac{d S}{d t}=\varphi_{0}-\mu S
$$

which has the solution

$$
S(t) \leq \frac{\varphi_{0}}{\mu}+\left(S(0)-\frac{\varphi_{0}}{\mu}\right) e^{-\mu t}
$$

Clearly, $S(t) \rightarrow \frac{\varphi_{0}}{\mu}$ as $t \rightarrow \infty$ regardless of the initial value $S(0)$. Therefore, it shows that condition $\mathrm{C}_{1}$ (of lemma 2) holds for our model.

Next, the right-hand side of the infectious subsystem (i.e. equations for $I_{1}, I_{2}, T, T_{A}$ and $A$ ) can be written as:

$$
\frac{d X_{2}}{d t}=G\left(X_{1}, X_{2}\right)=\left[\begin{array}{c}
-(\delta+\mu+\theta) I_{1}+\frac{1-u_{1}}{N} \beta_{1} c_{1} \frac{\varphi_{0}}{\mu} I_{1} \\
+\frac{1-u_{1}}{N} \beta_{2} c_{2} \frac{\varphi_{0}}{\mu} I_{2}+\frac{1-u_{1}}{N} \beta_{3} c_{3} \frac{\varphi_{0}}{\mu} T \\
+\frac{1-u_{1}}{N} \beta_{4} c_{4} \frac{\varphi_{0}}{\mu} T_{A}+\frac{1-u_{1}}{N} \beta_{5} c_{5} \frac{\varphi_{0}}{\mu} A \\
-\beta_{i} \frac{\varphi_{0}}{\mu}+\beta_{i} S \\
\theta I_{1}-\left(\delta+\mu+a_{1}\right) I_{2} \\
a_{1} I_{2}+a_{3} T_{A}-\left(\mu+\pi+a_{2}\right) T \\
a_{2} T-\left(\delta+\mu+a_{3}\right) T_{A} \\
\delta I_{1}+\delta I_{2}+\delta T_{A}+\pi T-(\mu+\alpha) A
\end{array}\right]
$$

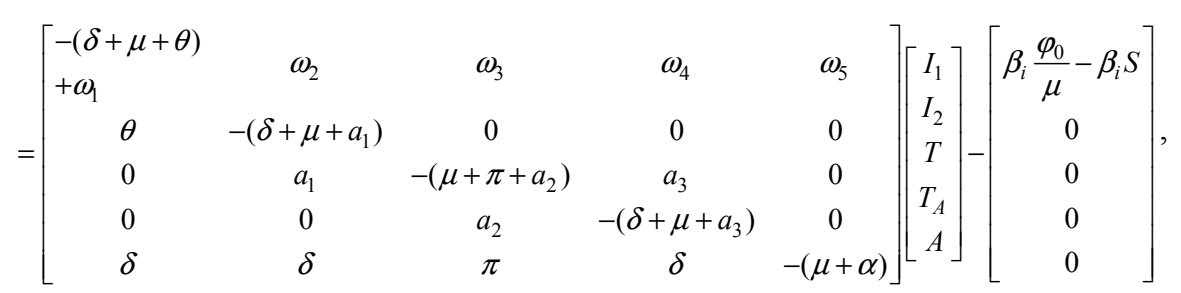

where $\omega_{1}=\frac{1-u_{1}}{N}\left(\beta_{1} c_{1} \frac{\varphi_{0}}{\mu}\right), \omega_{2}=\frac{1-u_{1}}{N}\left(\beta_{2} c_{2} \frac{\varphi_{0}}{\mu}\right), \omega_{3}=\frac{1-u_{1}}{N}\left(\beta_{3} c_{3} \frac{\varphi_{0}}{\mu}\right)$,

$$
\omega_{4}=\frac{1-u_{1}}{N}\left(\beta_{4} c_{4} \frac{\varphi_{0}}{\mu}\right) \text { and } \omega_{5}=\frac{1-u_{1}}{N}\left(\beta_{5} c_{5} \frac{\varphi_{0}}{\mu}\right)
$$

This implies that

$$
\frac{d X_{2}}{d t}=A X_{2}-\hat{G}\left(X_{1}, X_{2}\right)
$$

with

$$
A=\left[\begin{array}{ccccc}
-(\delta+\mu+\theta) & 1-u_{1}\left(\beta_{2} c_{2} \frac{\varphi_{0}}{\mu}\right) & \frac{1-u_{1}}{N}\left(\beta_{3} c_{3} \frac{\varphi_{0}}{\mu}\right) & \frac{1-u_{1}}{N}\left(\beta_{4} c_{4} \frac{\varphi_{0}}{\mu}\right) & \frac{1-u_{1}}{N}\left(\beta_{5} c_{5} \frac{\varphi_{0}}{\mu}\right) \\
+\frac{1-\varphi_{1}}{N}\left(\beta_{1} \frac{1}{\mu}\right) & \frac{\left.1-a_{1}\right)}{N} & 0 & 0 & 0 \\
\theta & -\left(\delta+\mu+a_{1}\right) & 0 \\
0 & a_{1} & -\left(\mu+\pi+a_{2}\right) & a_{3} & 0 \\
0 & 0 & a_{2} & -\left(\delta+\mu+a_{3}\right) & -(\mu+\alpha)
\end{array}\right]
$$

and

$$
\hat{G}\left(X_{1}, X_{2}\right)=\left[\begin{array}{c}
\beta_{i} \frac{\varphi_{0}}{\mu}-\beta_{i} S \\
0 \\
0 \\
0 \\
0
\end{array}\right]
$$

Then, it is obvious that $S \leq \frac{\varphi_{0}}{\mu}$, hence it is clear that $G(X, Y) \geq 0$ for all $(X, Y) \in \mathfrak{R}_{+}^{6}$. We also notice that the matrix $A$ is an $M$ - matrix, since its off-diagonal elements are non-negative. Hence, this proves the global stability of the $\operatorname{HAFE}\left(H^{0}\right)$.

\subsubsection{Global Stability of the HAEE}

Notably, the study of the endemic global stability is not only mathematically important, but also essential in predicting the evolution of the disease on the long run leading to prevention and intervention strategies. Achieving this goal, a number of methods in the range of monotone dynamical systems, the geometric approach and Lyapunov functions are used in conducting the global stability analysis of epidemic model, [22]. Here, to completely satisfy our aim of proving that the system HAEE for $\alpha=0$ is globally 
asymptotically stable, i.e., $R_{0}>1$, we shall adopt the later method - Lyapunov function with the incorporation of LaSalle's invariant principle, [29, 31].

In this regard, the following definitions and theorems (without proofs) are necessary:

Definition 2 (Lyapunov function)

A function $V(x)$ on a region $\Re_{D}$ of state space and containing an equilibrium point $X^{*}$ is called a Lyapunov function, if is satisfies the following conditions:

i. $V(x)$ is continuous and has continuous first order partial derivatives,

ii. $V(x)$ has a unique minimum at $X^{*}$ with respect to all other points in the region $\Re_{D}$,

iii. the function $V^{\prime}(x(t))=\bar{V} V(x) f(x) \quad$ satisfies $V^{\prime}(x(t)) \leq 0$ for all $x(t) \in \mathfrak{R}_{D}[23,32]$.
Theorem 7 If there exists a Lyapunov function for an equilibrium point $X^{*}$, then $X^{*}$ is a stable equilibrium point. If further, the function $V^{\prime}(x(t)) \leq 0$ for every point $x(t) \in \mathfrak{R}_{D}$, then the stability is asymptotic (Lyapunov, 1992).

Theorem 8 Let $x^{\prime}=f(x)$ be autonomous system. Suppose there is a neighborhood satisfies $X$ of 0 and continuously differentiable (time-independent) positive function $V: X \rightarrow \mathfrak{R}$, whose orbital derivative $V^{\prime}$ is negative semidefinite. Let $\tau$ be the union of all complete orbits contained in $\left\{x \in X \backslash V^{\prime}(x)=0\right\}$. Then, there is a neighborhood $U$ of 0 such that for every $x_{0} \in \omega\left(x_{0}\right) \leq \tau$ [31].

Now, let us recall our basic model (4) in its biological feasible domain

$$
\mathfrak{R}_{D}=\left\{\left(S, I_{1}, I_{2}, T, T_{A}, A\right) \in \mathfrak{R}_{+}^{6}: N=S(t)+I_{1}(t)+I_{2}(t)+T(t)+T_{A}(t)+A(t) \leq \frac{\varphi_{0}}{\mu}\right\},
$$

which is clearly a positively invariant set in $\Re^{6}$. Furthermore, since at the point $\alpha=0$, we see that $N=N^{*}=\frac{\varphi_{0}}{\mu}$ as $t \rightarrow \infty$, then we have the following limiting system:

$$
\left\{\begin{array}{l}
\frac{d S}{d t}=\varphi_{0}-\frac{1-u_{1}}{N^{*}}\left(\beta_{1} c_{1} I_{1}+\beta_{2} c_{2} I_{2}+\beta_{3} c_{3} T+\beta_{4} c_{4} T_{A}+\beta_{5} c_{5} A\right) S-\mu S, \\
\frac{d I_{1}}{d t}=\frac{1-u_{1}}{N^{*}}\left(\beta_{1} c_{1} I_{1}+\beta_{2} c_{2} I_{2}+\beta_{3} c_{3} T+\beta_{4} c_{4} T_{A}+\beta_{5} c_{5} A\right) S-(\mu+\delta+\theta) I_{1}, \\
\frac{d I_{2}}{d t}=\theta I_{1}-\left(\mu+\delta+a_{1}\right) I_{2}, \\
\frac{d T}{d t}=a_{1} I_{2}+a_{3} T_{A}-\left(\mu+\pi+a_{2}\right) T, \\
\frac{d T_{A}}{d t}=a_{2} T-\left(\mu+\delta+a_{3}\right) T_{A}, \\
\frac{d A}{d t}=\delta\left(I_{1}+I_{2}+T_{A}\right)+\pi T-\mu A .
\end{array}\right.
$$

To prove global stability result, we propose the following Lyapunov function

$$
V=w_{1}\left(S-S^{*}\right)^{2}+w_{2}\left(I_{1}-I_{1}^{*}\right)^{2}+w_{3}\left(I_{2}-I_{2}^{*}\right)^{2}+w_{4}\left(T-T^{*}\right)^{2}+w_{5}\left(T_{A}-T_{A}^{*}\right)^{2}+w_{6}\left(A-A^{*}\right)^{2},
$$

or

$$
V=\sum_{i=1}^{6} w_{i}\left(N_{i}-N_{i}^{*}\right)^{2}
$$

where $w_{i=i, \ldots, 6}>0$ are positive Lyapunov constants, $N_{i}$ is the population of $i^{\text {th }}$ compartment, $N_{i}^{*}$ is the equilibrium of $N_{i}$ and $V$, a continuous and differentiable function. Computing the time derivative of $V$ along the trajectories of the system (4), we obtain:

$$
\dot{V}=2 w_{1}\left(S-S^{*}\right) \dot{S}+2 w_{2}\left(I_{1}-I_{1}^{*}\right) \dot{I}_{1}+2 w_{3}\left(I_{2}-I_{2}^{*}\right) \dot{I}_{2}+2 w_{4}\left(T-T^{*}\right) \dot{T}+2 w_{5}\left(T_{A}-T_{A}^{*}\right) \dot{T}_{A}+2 w_{6}\left(A-A^{*}\right) \dot{A}
$$

Substituting the derivative of equation (29) into equation (31), we have, 


$$
\begin{aligned}
& \dot{V}=2 w_{1}\left(S-S^{*}\right)\left[\frac{1-u_{1}}{N^{*}}\left(\begin{array}{l}
-\beta_{1} c_{1}\left(I_{1} S-I_{1}^{*} S^{*}\right)-\beta_{2} c_{2}\left(I_{2} S-I_{2}^{*} S^{*}\right)-\beta_{3} c_{3}\left(T S-T^{*} S^{*}\right) \\
-\beta_{4} c_{4}\left(T_{A} S-T_{A}^{*} S^{*}\right)-\beta_{5} c_{5}\left(A S-A^{*} S^{*}\right)
\end{array}\right)-\mu\left(S-S^{*}\right)\right]
\end{aligned}
$$

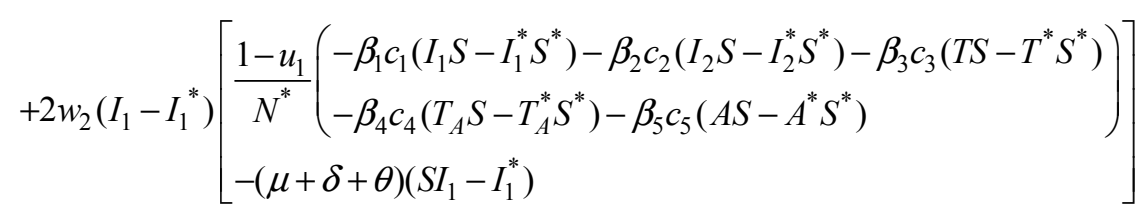

$$
\begin{aligned}
& +2 w_{3}\left(I_{2}-I_{2}^{*}\right)\left[\theta\left(I_{1}-I_{1}^{*}\right)-\left(\mu+\delta+a_{1}\right)\left(I_{2}-I_{2}^{*}\right)\right] \\
& +2 w_{4}\left(T-T^{*}\right)\left[a_{1}\left(I_{2}-I_{2}^{*}\right)+a_{3}\left(T_{A}-T_{A}^{*}\right)-\left(\mu+\pi+a_{2}\right)\left(T-T^{*}\right)\right] \\
& +2 w_{5}\left(T_{A}-T_{A}^{*}\right)\left[a_{2}\left(T-T^{*}\right)-\left(\mu+\delta+a_{3}\right)\left(T_{A}-T_{A}^{*}\right)\right] \\
& +2 w_{6}\left(A-A^{*}\right)\left[\delta\left(I_{1}-I_{1}^{*}\right)+\delta\left(I_{2}-I_{2}^{*}\right)+\delta\left(T_{A}-T_{A}^{*}\right)+\pi\left(T-T^{*}\right)-\mu\left(A-A^{*}\right)\right] .
\end{aligned}
$$

Then, we add the expression $\beta_{1} c_{1} I_{1} S^{*}, \beta_{2} c_{2} I_{2} S^{*}, \beta_{3} c_{3} T S^{*}, \beta_{4} c_{4} T_{A} S^{*}$ and $\beta_{5} c_{5} A S^{*}$ into the first and second square brackets to obtain the result

$$
\begin{aligned}
& \left.\dot{V}=2 w_{1}\left(S-S^{*}\right)\left[\begin{array}{l}
\frac{1-u_{1}}{N^{*}}\left(\begin{array}{l}
-\beta_{1} c_{1}\left(I_{1} S-I_{1}^{*} S^{*}\right)+\beta_{1} c_{1} I_{1} S^{*}-\beta_{1} c_{1} I_{1} S^{*}-\beta_{2} c_{2}\left(I_{2} S-I_{2}^{*} S^{*}\right) \\
+\beta_{2} c_{2} I_{2} S^{*}-\beta_{2} c_{2} I_{2} S^{*}-\beta_{3} c_{3}\left(T S-T^{*} S^{*}\right)+\beta_{3} c_{3} T S^{*}-\beta_{3} c_{3} T S^{*} \\
-\beta_{4} c_{4}\left(T_{A} S-T_{A}^{*} S^{*}\right)+\beta_{4} c_{4} T_{A} S^{*}-\beta_{4} c_{4} T_{A} S^{*} \\
-\beta_{5} c_{5}\left(A S-A^{*} S^{*}\right)+\beta_{5} c_{5} A S^{*}-\beta_{5} c_{5} A S^{*}
\end{array}\right. \\
-\mu\left(S-S^{*}\right)
\end{array}\right]\right) \\
& +2 w_{2}\left(I_{1}-I_{1}^{*}\right)\left[\begin{array}{l}
\frac{1-u_{1}}{N^{*}}\left(\begin{array}{l}
-\beta_{1} c_{1}\left(I_{1} S-I_{1}^{*} S^{*}\right)+\beta_{1} c_{1} I_{1} S^{*}-\beta_{1} c_{1} I_{1} S^{*}-\beta_{2} c_{2}\left(I_{2} S-I_{2}^{*} S^{*}\right) \\
+\beta_{2} c_{2} I_{2} S^{*}-\beta_{2} c_{2} I_{2} S^{*}-\beta_{3} c_{3}\left(T S-T^{*} S^{*}\right)+\beta_{3} c_{3} T S^{*}-\beta_{3} c_{3} T S^{*} \\
-\beta_{4} c_{4}\left(T_{A} S-T_{A}^{*} S^{*}\right)+\beta_{4} c_{4} T_{A} S^{*}-\beta_{4} c_{4} T_{A} S^{*} \\
-\beta_{5} c_{5}\left(A S-A^{*} S^{*}\right)+\beta_{5} c_{5} A S^{*}-\beta_{5} c_{5} A S^{*}
\end{array}\right. \\
-(\mu+\delta+\theta)\left(I_{1}-I_{1}^{*}\right)
\end{array}\right] \\
& +2 w_{3}\left(I_{2}-I_{2}^{*}\right)\left[\theta\left(I_{1}-I_{1}^{*}\right)-\left(\mu+\delta+a_{1}\right)\left(I_{2}-I_{2}^{*}\right)\right] \\
& +2 w_{4}\left(T-T^{*}\right)\left[a_{1}\left(I_{2}-I_{2}^{*}\right)+a_{3}\left(T_{A}-T_{A}^{*}\right)-\left(\mu+\pi+a_{2}\right)\left(T-T^{*}\right)\right] \\
& +2 w_{5}\left(T_{A}-T_{A}^{*}\right)\left[a_{2}\left(T-T^{*}\right)-\left(\mu+\delta+a_{3}\right)\left(T_{A}-T_{A}^{*}\right)\right] \\
& +2 w_{6}\left(A-A^{*}\right)\left[\delta\left(I_{1}-I_{1}^{*}\right)+\delta\left(I_{2}-I_{2}^{*}\right)+\delta\left(T_{A}-T_{A}^{*}\right)+\pi\left(T-T^{*}\right)-\mu\left(A-A^{*}\right)\right] .
\end{aligned}
$$

Simplifying equation (32), we have,

$$
\dot{V}=2 w_{1}\left(S-S^{*}\right)\left[\frac{1-u_{1}}{N^{*}}\left(\begin{array}{l}
-\beta_{1} c_{1} I_{1}\left(S-S^{*}\right)-\beta_{1} c_{1} S^{*}\left(I-I_{1}^{*}\right)-\beta_{2} c_{2} I_{2}\left(S-S^{*}\right) \\
-\beta_{2} c_{2} S^{*}\left(I_{2}-I_{2}^{*}\right)-\beta_{3} c_{3} T\left(S-S^{*}\right)-\beta_{3} c_{3} S^{*}\left(T-T^{*}\right) \\
-\beta_{4} c_{4} T_{A}\left(S-S^{*}\right)-\beta_{4} c_{4} S^{*}\left(T_{A}-T_{A}^{*}\right) \\
-\beta_{5} c_{5} A\left(S-S^{*}\right)-\beta_{5} c_{5} S^{*}\left(A-A^{*}\right)
\end{array}\right)-\mu\left(S-S^{*}\right)\right]
$$




$$
\begin{aligned}
& +2 w_{2}\left(I_{1}-I_{1}^{*}\right)\left[\frac{u_{1}}{N^{*}}\left(\begin{array}{l}
\beta_{1} c_{1} I_{1}\left(S-S^{*}\right)+\beta_{1} c_{1} S^{*}\left(I-I_{1}^{*}\right)+\beta_{2} c_{2} I_{2}\left(S-S^{*}\right) \\
+\beta_{2} c_{2} S^{*}\left(I_{2}-I_{2}^{*}\right)+\beta_{3} c_{3} T\left(S-S^{*}\right)+\beta_{3} c_{3} S^{*}\left(T-T^{*}\right) \\
+\beta_{4} c_{4} T_{A}\left(S-S^{*}\right)+\beta_{4} c_{4} S^{*}\left(T_{A}-T_{A}^{*}\right) \\
+\beta_{5} c_{5} A\left(S-S^{*}\right)+\beta_{5} c_{5} S^{*}\left(A-A^{*}\right)
\end{array}\right]+2 w_{3}\left(I_{2}-I_{2}^{*}\right)\left[\theta\left(I_{1}-I_{1}^{*}\right)-\left(\mu+\delta+a_{1}\right)\left(I_{2}-I_{2}^{*}\right)\right]\right. \\
& +2 w_{4}\left(T-T^{*}\right)\left[a_{1}\left(I_{2}-I_{2}^{*}\right)+a_{3}\left(T_{A}-T_{A}^{*}\right)-\left(\mu+\pi+a_{2}\right)\left(T-T^{*}\right)\right]+2 w_{5}\left(T_{A}-T_{A}^{*}\right)\left[a_{2}\left(T-T^{*}\right)-\left(\mu+\delta+a_{3}\right)\left(T_{A}-T_{A}^{*}\right)\right] \\
& +2 w_{6}\left(A-A^{*}\right)\left[\delta\left(I_{1}-I_{1}^{*}\right)+\delta\left(I_{2}-I_{2}^{*}\right)+\delta\left(T_{A}-T_{A}^{*}\right)+\pi\left(T-T^{*}\right)-\mu\left(A-A^{*}\right)\right] \\
& \dot{V}=2 w_{1}\left[\frac{1-u_{1}}{N^{*}}\left(-\beta_{1} c_{1} I_{1}-\beta_{2} c_{2} I_{2}-\beta_{3} c_{3} T-\beta_{4} c_{4} T_{A}-\beta_{5} c_{5} A\right)-\mu\right]\left(S-S^{*}\right)^{2} \\
& -2 w_{1} \frac{1-u_{1}}{N^{*}} \beta_{1} c_{1} S^{*}\left(S-S^{*}\right)\left(I-I_{1}^{*}\right)-2 w_{1} \frac{1-u_{1}}{N^{*}} \beta_{2} c_{2} S^{*}\left(S-S^{*}\right)\left(I_{2}-I_{2}^{*}\right) \\
& -2 w_{1} \frac{1-u_{1}}{N^{*}} \beta_{3} c_{3} S^{*}\left(S-S^{*}\right)\left(T-T^{*}\right)-2 w_{1} \frac{1-u_{1}}{N^{*}} \beta_{4} c_{4} S^{*}\left(S-S^{*}\right)\left(T_{A}-T_{A}^{*}\right) \\
& -2 w_{1} \frac{1-u_{1}}{N^{*}} \beta_{5} c_{5} S^{*}\left(S-S^{*}\right)\left(A-A^{*}\right) \\
& +2 w_{2}\left[\frac{1-u_{1}}{N^{*}} \beta_{1} c_{1} S^{*}-(\mu+\delta+\theta)\right]\left(I_{1}-I_{1}^{*}\right)^{2} \\
& +2 w_{2}\left[\frac{1-u_{1}}{N^{*}}\left(\beta_{1} c_{1} I_{1}+\beta_{2} c_{2} I_{2}+\beta_{3} c_{3} T+\beta_{4} c_{4} T_{A}+\beta_{5} c_{5} A\right)\right]\left(I-I_{1}^{*}\right)\left(S-S^{*}\right) \\
& +2 w_{2} \frac{1-u_{1}}{N^{*}} \beta_{2} c_{2} S^{*}\left(I-I_{1}^{*}\right)\left(I_{2}-I_{2}^{*}\right)+2 w_{2} \frac{1-u_{1}}{N^{*}} \beta_{3} c_{3} S^{*}\left(I-I_{1}^{*}\right)\left(T-T^{*}\right) \\
& +2 w_{2} \frac{1-u_{1}}{N^{*}} \beta_{4} c_{4} S^{*}\left(I-I_{1}^{*}\right)\left(T_{A}-T_{A}^{*}\right)+2 w_{2} \frac{1-u_{1}}{N^{*}} \beta_{5} c_{5} S^{*}\left(I-I_{1}^{*}\right)\left(A-A^{*}\right) \\
& +2 w_{3} \theta\left(I_{2}-I_{2}^{*}\right)\left(I_{1}-I_{1}^{*}\right)-2 w_{3}\left(\mu+\delta+a_{1}\right)\left(I_{2}-I_{2}^{*}\right)^{2} \\
& +2 w_{4} a_{1}\left(T-T^{*}\right)\left(I_{2}-I_{2}^{*}\right)+2 w_{4} a_{3}\left(T-T^{*}\right)\left(T_{A}-T_{A}^{*}\right) \\
& -2 w_{4}\left(\mu+\pi+a_{2}\right)\left(T-T^{*}\right)^{2}+2 w_{5} a_{2}\left(T_{A}-T_{A}{ }^{*}\right)\left(T-T^{*}\right)-2 w_{5}\left(\mu+\delta+a_{3}\right)\left(T_{A}-T_{A}{ }^{*}\right)^{2} \\
& +2 w_{6} \delta\left(A-A^{*}\right)\left(I_{1}-I_{1}^{*}\right)+2 w_{6} \delta\left(A-A^{*}\right)\left(I_{2}-I_{2}^{*}\right)+2 w_{6} \delta\left(A-A^{*}\right)\left(T_{A}-T_{A}^{*}\right) \\
& +2 w_{6} \pi\left(A-A^{*}\right)\left(T-T^{*}\right)-2 w_{6} \mu\left(A-A^{*}\right)^{2} \text {. }
\end{aligned}
$$

$$
\dot{V}=Q\left(Z Y+Y^{T} Z^{T}\right) Q^{T}
$$

with $Q=\left[S-S^{*}, I_{1}-I_{1}^{*}, I_{2}-I_{2}^{*}, T-T^{*}, T_{A}-T_{A}^{*}, A-A^{*}\right], Z=\operatorname{diag}\left(w_{1}, w_{2}, w_{3}, w_{4}, w_{5}, w_{6}\right)$ and

$$
Y=\left[\begin{array}{cccccc}
\lambda_{1} & -\frac{1-u_{1}}{N^{*}} \beta_{1} c_{1} S^{*} & \lambda_{4} & \lambda_{6} & \lambda_{8} & \lambda_{10} \\
\lambda_{2} & \lambda_{3} & \lambda_{5} & \lambda_{7} & \lambda_{9} & \lambda_{11} \\
0 & \theta & -\left(\mu+\delta+a_{1}\right) & 0 & 0 & 0 \\
0 & 0 & a_{1} & -\left(\mu+\pi+a_{2}\right) & a_{3} & 0 \\
0 & 0 & 0 & a_{2} & -\left(\mu+\delta+a_{3}\right) & 0 \\
0 & \delta & \delta & \pi & \delta & -\mu
\end{array}\right],
$$

where 


$$
\begin{aligned}
& \lambda_{1}=\frac{1-u_{1}}{N^{*}}\left(-\beta_{1} c_{1} I_{1}-\beta_{2} c_{2} I_{2}-\beta_{3} c_{3} T-\beta_{4} c_{4} T_{A}-\beta_{5} c_{5} A\right)-\mu, \\
& \lambda_{2}=\frac{1-u_{1}}{N^{*}}\left(\beta_{1} c_{1} I_{1}+\beta_{2} c_{2} I_{2}+\beta_{3} c_{3} T+\beta_{4} c_{4} T_{A}+\beta_{5} c_{5} A\right), \\
& \lambda_{3}=\frac{1-u_{1}}{N^{*}} \beta_{1} c_{1} S^{*}-(\mu+\delta+\theta), \quad \lambda_{4}=-\frac{1-u_{1}}{N^{*}} \beta_{2} c_{2} S^{*}, \quad \lambda_{5}=\frac{1-u_{1}}{N^{*}} \beta_{2} c_{2} S^{*}, \\
& \lambda_{6}=-\frac{1-u_{1}}{N^{*}} \beta_{3} c_{3} S^{*}, \quad \lambda_{7}=\frac{1-u_{1}}{N^{*}} \beta_{3} c_{3} S^{*}, \quad \lambda_{8}=-\frac{1-u_{1}}{N^{*}} \beta_{4} c_{4} S^{*}, \\
& \lambda_{9}=\frac{1-u_{1}}{N^{*}} \beta_{4} c_{4} S^{*}, \quad \lambda_{10}=-\frac{1-u_{1}}{N^{*}} \beta_{5} c_{5} S^{*} \text { and } \quad \lambda_{11}=\frac{1-u_{1}}{N^{*}} \beta_{5} c_{5} S^{*} .
\end{aligned}
$$

Then, to discuss the global asymptotic stability of $Q$, we have to show that the matrix $Y$ of equation (34) is Lyapunov stable or $-Y$ is diagonal stable. Thus, the following lemmas and theorem completely absorbed the required proof.

Lemma 4 For the matrix $Y$ defined by equation (34), let us consider $D=-Y$, and then $D$ is diagonal stable.

Lemma 5 For the matrix $Y$ defined by equation (34), the matrix $E=-Y^{-1}$ is diagonal stable.

We prove lemmas 4 and 5 using the following lemma:

Lemma 6 Let $D=\left[d_{i j}\right]$ be a non-singular $n \times n$ matrix $(n \geq 2)$ and $M=\operatorname{diag}\left(m_{1}, \ldots \ldots, m_{n}\right)$ be a positive diagonal $n \times n$ matrix. Let $E=D^{-1}$. Then, if $d_{n n}>0$, $\widetilde{M E}+(\widetilde{M E})^{T}>0$ and $\widetilde{M D}+(\widetilde{M D})^{T}>0$, it is possible to choose $m_{n}>0$ such that $\widetilde{M D}+D^{T} M^{T}>0$.

Hence, the following theorem holds.

Theorem 9 The matrix $Y$ defined by equation (34) is Lyapunov stable.

Proof

Based on lemmas 5 and 6 , and since $-Y_{66}>0$ i.e., $-(-\mu)>0$, there exists a positive diagonal matrix

$$
Z(-Y)+(-Y)^{T} Z^{T}>0 \text {. Thus, } Z Y+Y^{T} Z^{T}<0 .
$$

Therefore, using the LaSalle's invariant principle, the proof for the global stability of the system endemic equilibrium follows from the next theorem.

Theorem 10 The endemic equilibrium $H^{*}=\left(S^{*}, I_{1}^{*}, I_{2}^{*}, T^{*}, T_{A}^{*}, A^{*}\right)$ of model (4) is globally asymptotically stable in $H^{0}$ if $R_{0}>1$ and unstable otherwise.

Proof

Based on lemmas 5, 6 and theorem 9, we obtain $\frac{d V}{d t}<0$ when $H^{0} \neq H^{*}$ and $H^{0}$ is not on $S$-axis (a set of measure zero Therefore, the largest invariant set in $H^{0}$ such that $\frac{d V}{d t}=0$ is the singleton $H^{*}$, which is our endemic equilibrium point. Then, by LaSalle's invariant principle (Theorem 8), it follows that $H^{*}$ (endemic equilibrium of the system model (4)) is globally asymptotically stable (GAS) in $\mathfrak{R}_{D}$ if $H^{0} \neq H^{*}$. This complete the proof. $\square$

Now, having shown that our model state variables are all nonnegative with known stability behavioral pattern, it becomes paramount to numerically illustrate our theoretical predictions.

\section{Numerical Simulations and Results}

We devote this section to the simulations of derived system model and some key parameter variables to illustrate the ingenuity of our theoretical examination of system set goals. For simplicity and to completely exhaust insight into the intense of the study, the numerical simulations is considered in stepwise sequence for the derived model equations. That is, we first demonstrate our extended basic model (4) when both control functions are at zeros (i.e. $\left.u_{1}=0, \eta=\left(a_{1}+a_{3}\right)=0\right)$ and then the stability dynamics following onset application of control functions i.e. $u_{1}>0, \eta>0$. This is followed with the simulation of the system reproduction numbers under off-treatment scenario, i.e. $R_{0}=R_{0(1)}$ and when onset-treatment was observed i.e., $R_{0}=R_{0(2)}$. The essence is to explicitly define the impact of application of global stability theory on the role of ART abuse in the treatment dynamics of HIV/AIDS infection. Notably, the entire simulations are computed using an in-built rkfixed function of Runge-Kutta of order of precision 4 in a Mathcad surface. To this effect, we generate system data from verified models as depicted by (Table 1) below, [33-37].

Table 1. Variables, parameters and their values for model (4).

\begin{tabular}{llll}
\hline \multirow{2}{*}{ Variables } & Dependent State variables & Initial Values & Units \\
\cline { 2 - 3 } & Description & 0.5 & \\
\hline$S(t)$ & Susceptible population & 0.1 & $\mathrm{cells} / \mathrm{mm}^{3}$ \\
$I_{1}(t)$ & Unaware infectives & 0.1 & \\
$I_{2}(t)$ & Aware infectives but not receiving treatment & 0.1 & \\
$T(t)$ & Aware infectives receiving treatment & \\
\hline
\end{tabular}




\begin{tabular}{llll}
\hline Variables & Dependent State variables & Initial Values & Units \\
\cline { 2 - 3 }$A(t)$ & Description & 0.1 & \\
& Aware infectives under ART abuse & 0.1 & $\mathrm{~mm}^{3} \mathrm{~d}^{-1}$ \\
& Full-blown AIDS population & & $\mathrm{mm}^{3} \mathrm{~d}^{-1}$ \\
$T_{0}(t)$ & Parameters and Constants & 0.5 & $\mathrm{~mm}^{3} \mathrm{vir}^{-1} \mathrm{~d}^{-1}$ \\
$\beta_{i=1, \ldots, 5}$ & Description & Recruitment rate (population source) & $0.32 ; 0.27 ; 0.175 ; 0.125 ; 0.05$ \\
$c_{i=1, \ldots, 5}$ & Probability of interaction by susceptible with various infectives & $0.5 ; 0.4 ; 0.3 ; 0.2 ; 0.1$ \\
$\lambda$ & Sexual contact by susceptible with various infectives & $u_{1} \in[0,1]$ & $\mathrm{day}^{-1}$ \\
$\theta$ & Successful condom use & 0.04 & \\
$\pi$ & Rate at which the unaware becomes aware & 0.028 & $\mathrm{~mm}^{3} \mathrm{~d}^{-1}$ \\
$\delta$ & Progression rate of aware infective to AIDS & 0.5 & \\
$\alpha$ & Rate at which infectives develops AIDS & 0.3 \\
$\mu$ & AIDS induce death rate & 0.002 \\
$a_{1}$ & Natural death rate & 0.45 \\
$a_{2}$ & Rate of ART receive by $T(t)$ & 0.37 \\
$a_{3}$ & Rate of abuse of ART by $T_{A}(t)$ & 0.14 \\
\hline
\end{tabular}

Note: Table 1, is a reflection of verified data modified to accommodate the present study [33-37].

\subsection{Simulation of System Basic Model (Without Control Functions)}

We recall that our goal is to investigate the application of global stability analysis of the role of ART abuse on multitreatment functions (condom use and ART). That is, we simulate as leverage to system set goal, the system derived basic model equation (4) in a completely off-treatment scenario (i.e., $u_{1}=0, a_{1}=0=a_{3}$ ). In reality, the essence of this simulation is to allow us ascertain the magnitude of HIV/AIDS infection transmission dynamics at off-treatment and when compared to the accessibility of control functions.

Therefore, invoking basic model (4), for all $u_{1}=0, a_{1}=0=a_{3}$ and recalling (Table 1), we simulate the six subgroups from which the following results are derived as depicted by Figure 2(a-f) below:

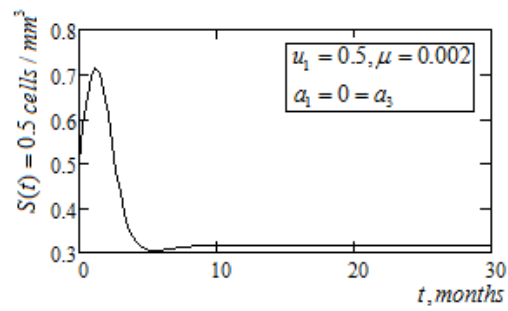

a) Dynamics of susceptible population under off-treatment $\varphi_{0}=0.5 \mathrm{~mm}^{3} \mathrm{~d}^{-1}$

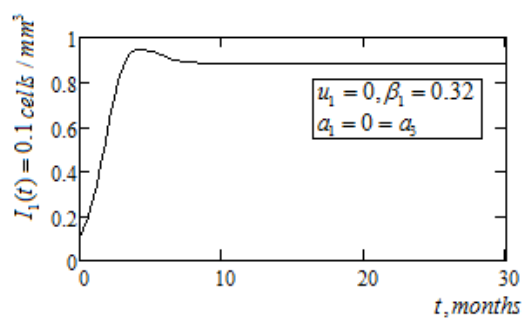

b) Dynamics of unaware infective under off-treatment $\mu=0.02 d^{-1}$

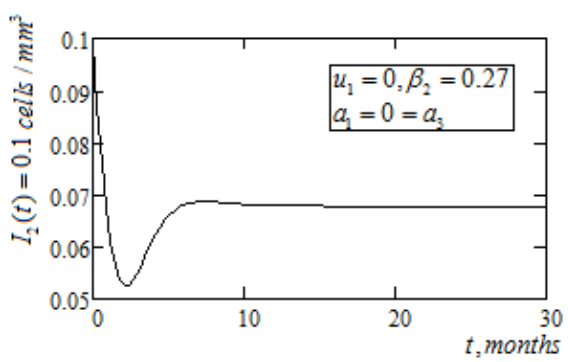

c) Dynamics of aware infective not ready for treatment under off-treatment $\mu=0.02 d^{-1}$

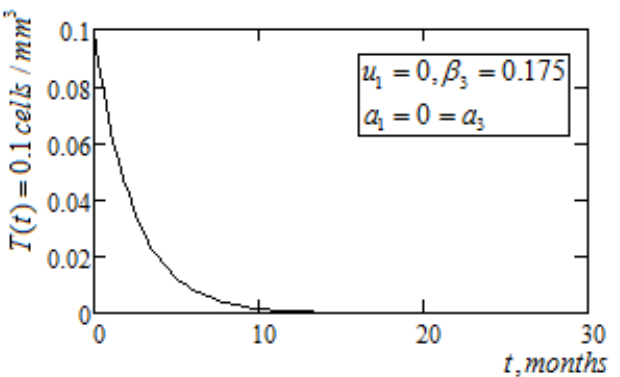

d) Dynamics of aware infective on treatment under off-treatment $\mu=0.02 d^{-1}$

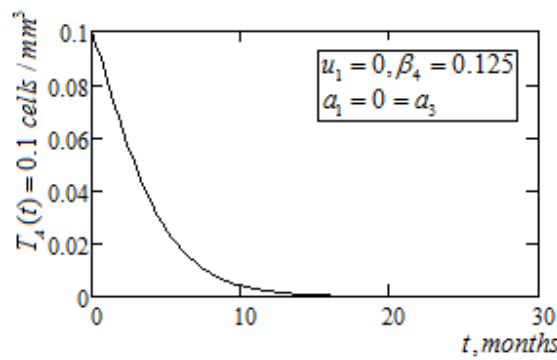

e) Dynamics of aware infective with ART abuse under off-treatment $\mu=0.02 d^{-1}$ 


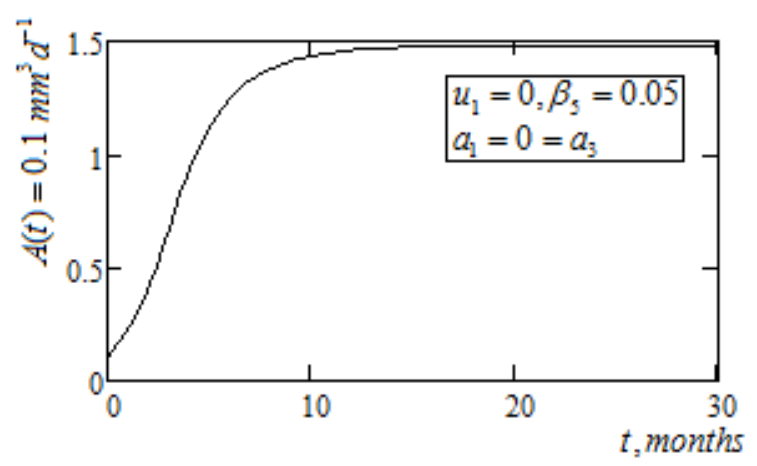

f) Dynamics of full-blown AIDS infection under off-treatment

$$
\alpha=0.3 \mathrm{~mm}^{3} d^{-1}
$$

Figure 2. (a-f). Schematic representation of off-treatment of HIV/AIDS dynamics under of ART abuse scenario with $R_{0(1)}=0.343$

Obviously, Figure 2(a-f) represents the dynamical flow of HIV/AIDS transmission with ART abuse and in particular under off-treatment scenario. Notably, Figure 2(a) depicts an undulating declination of the susceptible population with value of $0.5 \leq S(t) \leq 0.305$ cells $/ \mathrm{mm}^{3}$ at $t_{f} \leq 30$ months. Moreso, this decline in the susceptible population is seen in the upsurge of the unaware infected population attaining stability with value range of $0.1 \leq I_{1}(t) \leq 0.948 \mathrm{cells} / \mathrm{mm}^{3}$ at $t_{f} \leq 30$ months - see Figure 2(b). Figure 2(c) represent the screened infective that become aware of their HIV status, which exhibited some significant behavioral attitude that resulted to early stability after initial decline in population with varying range of $0.1 \leq I_{2}(t) \leq 0.053 \mathrm{cells} / \mathrm{mm}^{3}$ at $t_{f} \leq 3$ months and then to $I_{2}(t) \leq 0.07$ for all $3 \leq t_{f} \leq 30$ months.

From Figures 2(d \& e), we see the aware infectives that are on treatment compartments but have no access to treatment (i.e. off-treatment) exhibiting sequential near population extinctions leaving their varying population at $0.1 \leq T(t) \leq 0.581 \times 10^{-7}$ for all $12 \leq t_{f} \leq 30$ and $0.1 \leq T_{A}(t) \leq 0.255 \times 10^{-6}$ for all $16 \leq t_{f} \leq 30$ months respectively. Finally, the depletion in the susceptible population and the increasing population of the unaware infectives is seen to transmute rapidly to full-blown AIDS as vindicated by Figure 2(f) with value at $0.1 \leq A(t) \leq 1.483$ for all $t_{f} \leq 30$ months.

\subsection{Simulation of System Endemic Equilibria (with Onset-treatment Control Functions)}

To improve on the outcome of sub-section 5.1, the study introduced two control functions (condom use and ART) with $u_{1}=0.5, a_{1}=0.45, a_{3}=0.14$, allowing other parameters as in (Table 1) unchanged. Thus, we verify the model by simulating the system endemic equilibria under bilinear control functions with $R_{0}=R_{0(2)}=0.271$ as follows:

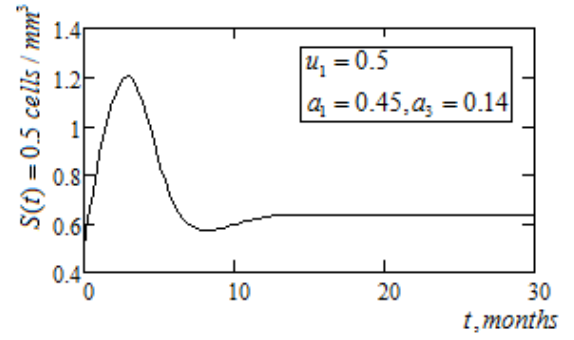

a) Dynamics of susceptible population under initial treatment with $\varphi_{0}=0.5 \mathrm{~mm}^{3} d^{-1}$

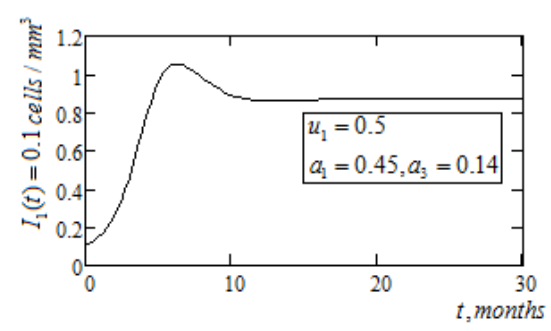

b) Dynamics of unaware infective under initial treatment with $\beta_{1}=0.32 \mathrm{~mm}^{3} \operatorname{vir}^{-1} d^{-1}$

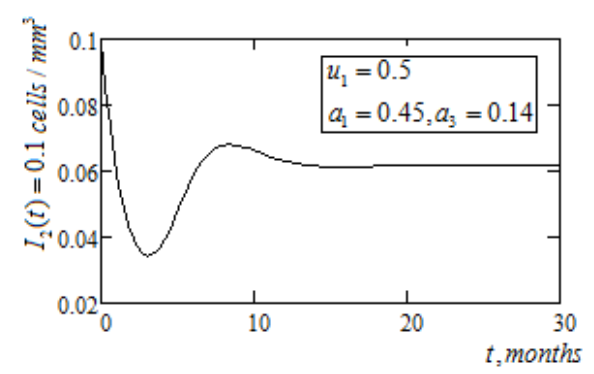

c) Dynamics of aware infective not ready for treatment but under initial treatment with $\beta_{2}=0.27 \mathrm{~mm}^{3}$ vir $^{-1} d^{-1}$

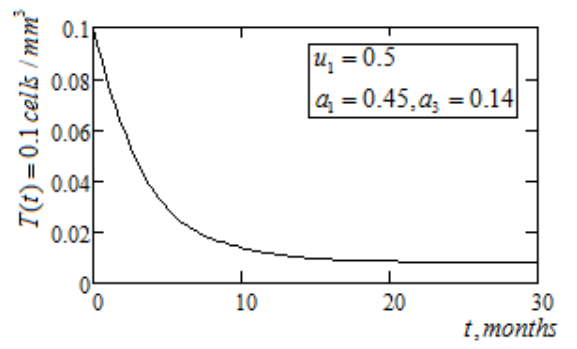

d) Dynamics of aware infective on treatment under initial treatment with $\beta_{3}=0.175 \mathrm{~mm}^{3}$ vir $^{-1} d^{-1}$

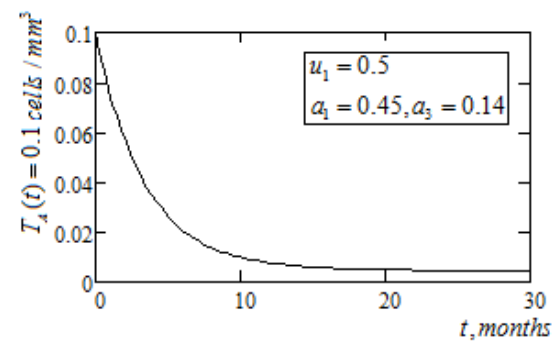

e) Dynamics of aware infective with ART abuse under initial treatment with $\beta_{4}=0.125 \mathrm{~mm}^{3}$ vir $^{-1} d^{-1}$ 


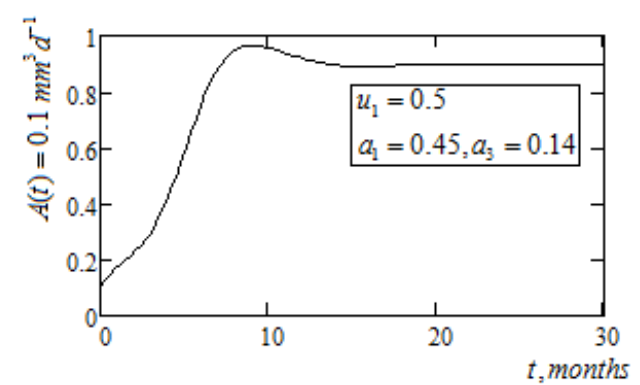

f) Dynamics of full-blown AIDS infection under initial treatment with

$$
\beta_{5}=0.05 \mathrm{~mm}^{3} \mathrm{vir}^{-1} d^{-1}
$$

Figure 3. $(a-f)$. Schematic representation of HIV/AIDS endemic model with onset-treatment under $A R T$ abuse scenario and $R_{0(2)}=0.271$

Figure. 3(a-f) above portrait the administration of onsettreatment in the dynamic flow of HIV/AIDS infection under ART abuse. Precisely, from Figure 3(a), we observe initial spontaneous increase in the susceptible population with value $0.5 \leq S(t) \leq 1.203$ cells $/ \mathrm{mm}^{3}$ for $t_{f} \leq 3$ months and then decline to initial value of $S(t) \leq 0.5 \mathrm{cells} / \mathrm{mm}^{3}$ at $t_{f} \leq 8$ months. The susceptible population then attain gradual stability of $S(t) \leq 0.62$ cells $/ \mathrm{mm}^{3}$ at $12 \leq t_{f} \leq 30$ months. Notably, the undulating curve of Figure 3(a) is reciprocated by the rapid initial inclination of infection for the unaware infectives with peak value at $0.1 \leq I_{1}(t) \leq 1.054$ cells $/ \mathrm{mm}^{3}$ for all $t_{f} \leq 7$ months. The dynamics of this subgroup exhibit slight decline with relative stability value of $I_{1}(t) \leq 0.85$ cells $/ \mathrm{mm}^{3}$ for all $10 \leq t_{f} \leq 30$ months - see Figure $3(\mathrm{~b})$. The aware infection not ready for treatment depicted by Figure 3(c) exhibit initial decline in the range of $0.1 \leq I_{2}(t) \leq 0.034$ cells $/ \mathrm{mm}^{3}$ at $t_{f} \leq 3$ months, only to surge with relative stability value $I_{2}(t) \leq 0.62 \mathrm{cells} / \mathrm{mm}^{3}$ for all $10 \leq t_{f} \leq 30$ months.

Interestingly, the compartment designating the aware infective under onset-treatment as depicted by Figure 3(d), shows tremendous decline in the rate of aware infectives with value $8.186 \times 10^{-3} \leq T(t) \leq 0.1$ cells $/ \mathrm{mm}^{3}$ for all $t_{f} \leq 10$ months and thereafter attain gradual stability all through $12 \leq t_{f} \leq 30$ months. For the aware infectives on initial onset-treatment with drug abuse tendency as depicted by Figure 3(e), the curve shows similar dynamics to that of Figure 3(d) but with intense decline through $t_{f} \leq 10$ following later resumption of treatment with diminishing range of $4.598 \times 10^{-3} \leq T_{A}(t) \leq 0.1 \quad$ cells $/ \mathrm{mm}^{3}$ from compartment $T_{A}(t)$ to compartment $T(t)$ for all $22 \leq t_{f} \leq 30$ months. On the other hand, the manifestation of the ART abuse is seen by the relatively persistence of the virus in the incline rate of full-blown AIDS population with stability trend of $0.85 \leq A(t) \leq 0.966$ cells $/ \mathrm{mm}^{3}$ for all $10 \leq t_{f} \leq 30$ months.
Thus, from the simulations of model (4) at off-treatment and at endemic stability equilibria for onset-treatment dynamics, it is obviously that the respective rate of infection transmission is a function of the system reproduction numbers. Therefore, our study will be biologically more meaningful, if the numerical simulations of these respective reproduction numbers are analyzed.

\subsection{Numerical Simulations of System Reproduction Numbers $\left(\boldsymbol{R}_{0(1)}, \boldsymbol{R}_{0(2)}\right)$}

Of note, in subsection 4.2, we had defined and evaluated the system reproduction number for both the off-treatment and onset-treatment scenarios. To further ascertain the impact and relationship of these varying reproduction number to our study, we simulate the reproduction number for off-treatment $R_{0(1)}$ and the reproduction number for onset-treatment $R_{0(2)}$ as seen below:

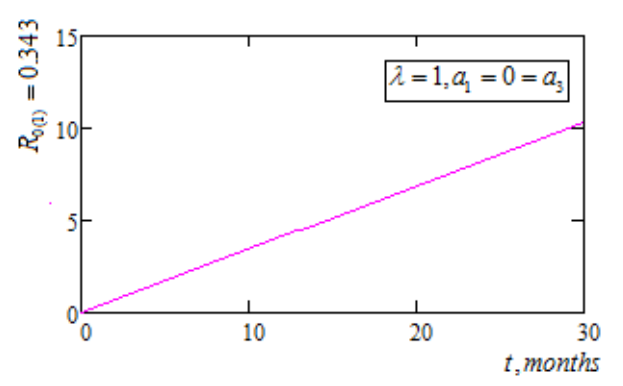

a) Basic reproduction number for off-treatment with $W_{1}=0, W_{2}=0$

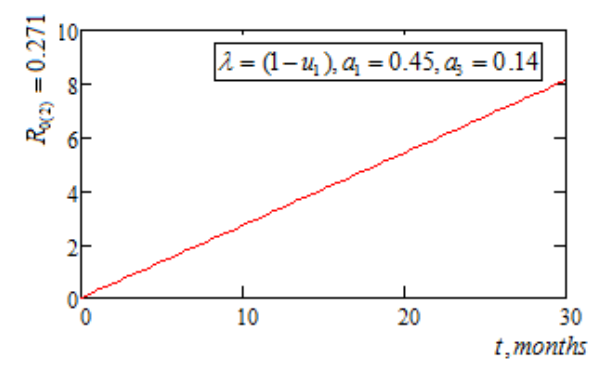

b) Basic reproduction number for onset-treatment with $W_{1}=0, W_{2}=0$

Figure 4. $(a \& b)$. Comparison of system reproduction numbers for offtreatment and onset-treatment scenarios.

From Figure 4(a \& b), it is explicitly obvious that at offtreatment scenario, the reproduction number with initial value $R_{0}=R_{0(1)}=0.343$ is seen to snowball to $R_{0(1)}=10.298$, through $t_{f} \leq 30$ months - see Figure 4(a). On the other hand, following the initiation of multiple control functions, it is observe that the reproduction number $\left(R_{0}=R_{0(2)}=0.271\right)$ for the system (4) with accessibility to treatment but truncated following ART abuse, incline to value $R_{0(2)}=7.806$ for all $t_{f} \leq 30$ months - see Figure $4(\mathrm{~b})$. The implication were that under off-treatment scenario, the spread of the virus is bound to escalate to an endemic proportion as to when compared to the situation where infection could be within controllable proportion following accessibility of 
control functions though truncated by abuse of therapy.

\section{Discussion of Results}

In this study, two compactible non-linear models have been extended and analyzed to study the role of antiretroviral therapy (ART) abuse for the treatment of HIV/AIDS epidemic, [21, 22]. That is, the present study sought to determine the mathematical importance in predicting the evolution of HIV/AIDS, which would lead to prevention and intervention strategy in the presence of ART abuse.

Of note, the main objective of the study is the application of global stability theory for the assessment of the role of ART abuse for the treatment of HIV/AIDS epidemic. Moreso, in addition to existing assumptions of motivating factors the main assumptions of the present study was the reclassification and incorporation of the aware infectives not ready to receive ART and those that are aware but with truncated (incoherent) treatment schedule. Also, it was assumed that the resumption rate for the use of ART is less compared to those that use ART and stop completely (abuse) the therapy (i.e. $a_{3}<a_{1}$ ).

From the material and methods adopted for this study, the entire investigation involved quantitative formulation of untreated 6-Dimensional ODE model consisting of interaction between susceptible, unaware infective, aware infectives not on treatment, aware infectives on treatment schedule, aware infectives with ART abuse and the fullblown AIDS population. The mathematical well-posedness of the basic model was investigated and existence of model equilibria established. Furthermore, quantitative analyzes of model was extended by using the next generation operator approach to compute the system reproduction number, which was defined in terms of off-treatment $R_{0(1)}$ and reproduction number for onset-treatment denoted by $R_{0(2)}$. It is founded that the model has two equilibria: HIV/AIDS free equilibrium (HAFE), which was locally asymptotically stable whenever $R_{0}<1$ and unstable whenever $R_{0}>1$, resulting to endemic equilibrium (HAEE). Global stability analysis of model (4) was determined using classical method of Lyapunov function in conjunction with the LaSalle's invariant principle. It was also established noting that if the basic reproduction number $R_{0}>1$, the unique endemic equilibrium is globally asymptotically stable.

In line with study set goal, a number of numerical simulations were performed. First, the simulation was considered for an off-treatment scenario with infectives and full-blown AIDS population inclined to an alarming proportion as evident by the near population extinction of the susceptible. An investigation that agrees with those of existing models for untreated HIV infection transmission dynamics, [36, 38]. Furthermore, the model was simulated following the application of onset-treatment devoid of optimal control approach with which the results clearly showed some significant departure when compared with those of off-treatment situation. That is, though infection was not completely eliminated, there was considerable reduction in the level and spread of the infection. This low-moderate outcome can be attributed to the significant abuse of ART by the aware infective that exhibited incoherent application of control functions. None-the-less, the present result is rather an improvement against system motivating models, where abuse of ART were not identified and given the desired attention [21, 22]. Notably, the severity of virions spread as indicated by aware infectives with ART abuse under offtreatment was rapid and high, reducing the population to $0.1 \leq T_{A}(t) \leq 1.255 \times 10^{-6}$ cells $/ \mathrm{mm}^{3}$ when compared to the rate of infection proportion under onset application of control functions with $0.1 \leq T_{A}(t) \leq 4.598 \times 10^{-3} \mathrm{cells} / \mathrm{mm}^{3}$. That is, under off-treatment, we observed near population extinction for $T_{A}(t)$. The corresponding susceptible population for these two simulations stood at $0.5 \leq S(t) \leq 0.35 \mathrm{cells} / \mathrm{mm}^{3}$ (decline in population) for off-treatment as against the value of $0.5 \leq S(t) \leq 1.203$ cells $/ \mathrm{mm}^{3}$ for the onset-treatment (relatively inclined population).

Furthermore, the varying outcomes from off-treatment and onset-treatment scenarios was affirmed by the simulations of the system reproduction numbers for off-treatment, which stood at $R_{0(1)}=0.343<1$ and for onset-treatment computed as $R_{0(2)}=0.271<1$. The implication are that the disease-free equilibrium for our model is locally asymptotically stable (LAS) with low infection transmission rate for onsettreatment as against high infection transmission rate when compared to off-treatment schedule. In comparative terms, the present results in relation to those of our motivating models proved that our control does behave somewhat differently from drug used to control systems not explicitly modeling the role ART abuse, [21, 22].

\section{Conclusion}

Motivated by unaccounted consequential role of ART abuse in the transmission and treatment of HIV/AIDS epidemic, the present study have been formulated to explicitly access the mathematical modeling of the role of ART abuse for the treatment of HIV/AIDS epidemic using global stability analysis. Giving an insight to the study, a 6dimenional deterministic non-linear mathematical model was derived and analyzed quantitatively.

With the incorporation of three infectious compartments to those of study motivating models, the study material and methods was quantitatively explored following the derivation of the system basic model and the system reproduction number for off/on treatment schedules, which was analytically investigated using next generation matrix approach. Furthermore, using the linearization process, we investigated the local stability conditions for HAFE and established the existence HAEE provided $R_{0}>1$. Moreso, classical Lyapunov function in conjunction with LaSalle's invariant principle was explored for the analytical investigation of the system global stability conditions in relation to its global asymptotic endemic equilibrium. The 
study then conducted numerical simulations sequel to derived model equations and key parameter variables to numerically investigate the validity of the application of global stability conditions. Discussion following simulated results were exhaustively examined. Results showed that the system reproduction number, which defined the varying rate of infection transmission was determined for both off/ontreatment. Clearly, $R_{0(1)}$ depicting off-treatment though less than 1 , was relatively higher when compared to reproduction number at onset-treatment schedule $R_{0(2)}$. Notably, the onsettreatment reproduction number for the present investigation ( $R_{0}=0.271<1$ ), was by far an improvement to the reproduction number computed by our motivating model with $R_{0}=0.903<1$, which is attributed to the onset identification and isolation of aware infectives with drug abuse clinical records. Furthermore, for off-treatment scenario, there exists rapid spread of infection leading to near extinction of the susceptible population. Moreso, application of onset-treatment functions with trend of ART abuse yield tremendous reduction in HIV/AIDS infection epidemic following the recovery rate of $0.5 \leq S(t) \leq 1.203$ cells $/ \mathrm{mm}^{3}$ at $t_{f} \leq 3$ months for susceptible population with attained stability of $S(t) \leq 0.62$ cells $/ \mathrm{mm}^{3}$ through $20 \leq t_{f} \leq 30$ months. This later decline is evidently a function of therapy (ART) abuse.

Conspicuously, the result does not only demonstrated the role of ART abuse but by far depicts an improved outcome when compared to outcome of onset-treatment schedule from those of study motivating models. More visibly, is the fact that the controls of the present study does behaved somewhat differently from drugs used to control systems not explicitly modeling ART abuse. Thus, the present study have explicitly shown the overwhelming significant impact of global stability analysis for the control/treatment of HIV/AIDS under therapy abuse scenario. Nonetheless, for enhanced maximization of susceptible population, it is highly suggested that the study could be further subjected to classical application of optimal control strategy and its optimal conditions for optimum control of HIV/AIDS infection under ART abuse.

\section{Acknowledgements}

The authors expressed their deep appreciation to their anonymous reviewers whose scientific and methodological suggestions elevated the standard of the final output of this article.

\section{Authors Contribution}

Bassey Echeng Bassey: Conceptualization, formulation, funding, writing, editing, review, programing and analysis. Adagba Odey Henry: Supervision, methodology, editing, and validation.

\section{Declaration of Competing Interest}

All the authors do not have any possible conflicts of interest.

\section{References}

[1] UNAIDS (2019). Fact Sheet - World Aids Day 2019, (On-line: https://www.firsnet.org/images/worlddays/World_AIDS_Day _Fact_Sheet.pdf)

[2] Ouattara, D. A. (2005). Mathematical Analysis of the HIV-1 Infection: Parameter Estimation, Therapies Effectiveness and Therapeutical Failures. Proceedings of the 2005 IEEE, Engineering in Medicine and Biology, 27th Annual Conference Shanghai, China, September 1-4.

[3] Seyed, M. M., Abba B. G., Robert G. M. and Richard G. (2003). Could condom stop the AIDS epidemic?, Theor. Med., 5 (3-4), 171-181.

[4] Mukandavire, Z., Garira W., Tchuenche, J. M. (2009). Modelling effects of public-health educational campaigns on HIV/AIDS transmission dynamics, Applied Mathematical Modelling 33, 1-5.

[5] Anderson, R. M. and May, R. M. (1991). Infectious Diseases of Humans: Dynamics and Control. Oxford University Press, N.Y., 290-292.

[6] Greenhalgh, D., Doyle, M. and Lewis, F. (2001). Mathematical treatment of AIDS and condom use, IMA. J. Math. Appl. Med. Biol., 18, 225-262.

[7] Hyman, J. M. and Stanley E. N. (2003). Modeling the impact of random screening and contact tracing in reducing the spread of HIV, Math. Biosc., 181, 17-54.

[8] Anderson, R. M., May, R. M., Medley, G. F. and Johnson, A. (1986). A preliminary study of the transmission of the human immunodeficiency virus (HIV), the causative agent of AIDS. IMA J. Math. Appl. Med. Biol., 3, 229-263.

[9] Knox, E. R. (1986). A transmission model for AIDS. European J. Epidemiology, 2, 165-177.

[10] May, R. M. and Anderson, R. M. (1987). Transmission dynamics of HIV infection. Nature, 3426, 137-142.

[11] Hsieh, H. Y. and Valasco-Hernandez, J. X. (1991). Modeling the effect of treatment and behavioral change in HIV transmission dynamics. (On-line: https://ecommons.cornell.edu/bitstream/handle/1813/31721/BU1143M.pdf\%3Bjsessionid\%3D0053CF3ED9DA07868A1BBB4 D92321815?sequence\%3D1

[12] Moghadas, S. M., Gumel, A. B., Mcleod, R. G. and Gordon, R. (2003). Could condom stop the AIDS epidemic? Journal of Theoretical Medicine, 5 (3-4), 171-181.

[13] Kimbir, A. R., Musa, S. and Bassey, E. B. (2006). On a twosex Mathematical model for the prevention of HIV/AIDS in a varying population. J. Math. Assoc. Nig., 33 (201), 1-13.

[14] Bassey, E. B. and Lebedev, K. A. (2015a). On mathematical model of the impact of non-compliance with preventive measures for the prevention of the spread of HIV/AIDS among heterosexual population. Scientific Journal of KubSAU, 108 (04), 1-8. 
[15] Kefale, B. and Kefale, Y. (2013). Knowledge, attitude, practice and determinant of condom use among people living with HIV/AIDS in Gondar University Hospital North West Ethiopia. J. Phys Pharm Adv, 3 (10), 247-260.

[16] Bracher, M., Santon G., Watkins, S. C. (2004). Assessing the potential of condom use to prevent the spread of HIV: a micro-simulation study. Stud. Fam. Plan., 35 (1), 48-64.

[17] Agha, S. (1998). Sexuality activity and condom use in Lusaka, Zambia. https://www.guttmacher.org/pubs/journals2403298.html)

[18] Bassey B. E. and Lebedev K. A. (2015b). On mathematical model of the impact of heterosexual use of condom and antiretroviral therapy for the prevention of HIV/AIDS epidemic, International Journal of Applied and Fundamental Research, 1 (2015), 1-17.

[19] Kimbir, A. R. and Oluwole, H. K. (2008). A mathematical model of HIV transmission dynamics considering counseling and antiretroviral therapy (ART). J. Mod. Math. Stat., 2 (5), 166-169.

[20] Bassey, B. E. and Lebedev, K. A. (2016). On numerical analysis of the impact of condom use and counseling for the prevention of heterosexual transmission of HIV/AIDS infection// Proceedings of the XXII-th international scientific conference "the Potential of modern science (Russian Federation, Lipetsk, March 28, 2016.) / Edited by M. J. Levin. - Lipetsk: "maximum information technology", 2 (19), 7-21. http://elibrary.ru/author_items.asp?authorid=80 1157

[21] Tripathi, A., Naresh, R. and Sharma, D. (2007). Modelling the effect screening of unaware infectives on the spread of HIV infection. Applied Mathematics and Computation 184 (2007) 1053-1068

[22] Zahedi, M. S. and Kargar, N. S. (2017). The VolterraLyapunov matrix theory for global stability analysis of a model of the HIV/AIDS. Int. J. Biomath., 10 (1): 1-21.

[23] Osman, S., Makinde, O. D. and Theuri, D. M. (2018). Stability analysis and modeling of listeriosis dynamics in human and animal populations. Global Journal of Pure and Applied Mathematics, 14 (1), 115-138.

[24] Mafuta, P., Mushanyu, J. and Nhawu, G. (2014). Invariant Region, Endemic Equilibria and Stability Analysis. IOSR Journal of Mathematics, 10 (2), 118-120.

[25] Akanni, J. O. and Akinpelu, F. O. (2016). Sensitivity analysis of HIV/AIDS model with vertical transmission, treatment and progression rate. International Journal of Scientific and Engineering Research, 7 (8), 497-514.

[26] Edward, S. and Nyerere, N. (2015). A Mathematical Model for the Dynamics of Cholera with Control Measures. Applied and Computational Mathematics, 4 (2), 53-63.

[27] Van den Driessche, P. and Watmough, J. (2002). Reproduction numbers and sub-threshold endemic equilibria for compartmental models of disease transmission. Mathematical Biosciences, 180 (2002), 29-48.

[28] Simon, C. P. and Blume, L. (1994). Mathematics for Economists. New York, W. W. Norton.

[29] Kahuru, J., Luboobi, L. and Nkansah-Gyekye, Y. (2017a). Stability analysis of the dynamics of tungiasis transmission in endemic areas. Asian Journal of Mathematics and Applications, 2017, 1-24.

[30] Castillo-Chavez, C., Feng, Z. and Huang, W. (2002). On the computation of $\mathrm{R}_{0}$ and its role on global stability in Mathematical Approaches for Emerging and Re-emerging Infectious Disease: An Introduction. IMA Volumes in Mathematical and its Approaches, 125, Springer, 229-250.

[31] LaSalle, J. P. (1976). The stability of dynamical systems. CBMS-NSF regional conference series in applied mathematics 25. SIAM, Philadelphia.

[32] Lyapunov, A. M. (1992). The General Problem of the Stability of motion, (A. T. Fuller trans.) Taylor and Francis, London.

[33] Hattaf, K. and Yousfi, N. (2012a). Two optimal treatments of HIV infection model. World Journal of Modeling and Simulation, 8 (1), 27-35.

[34] Culshaw, R. V., Ruan, S. and Spiteri, R. J. (2004). Optimal HIV treatment by maximizing immune response, Journal of Mathematical Biology, 48 (5), 545-562.

[35] Bassey, E. B. (2018). Dynamic optimal control model for dual-pair treatment functions of dual-delayed HIV-pathogen infections. Journal of Mathematical Sciences: Advances and Applications, 51 (1), 1-50.

[36] Zarei, H., Kamyad, A. V. and Effati, S. (2010). Maximizing of asymptomatic stage of fast progressive HIV infected patient using embedding methods. Intelligent Control and Automation, 1 (1), 48-58.

[37] Hattaf, K. and Yousfi, N. (2012b). Optimal control of a delayed HIV infection model with immune response using an efficient numerical method. ISRN Biomathematics, dio: $10.5402 / 2012 / 215124,1-7$.

[38] Landi, A., Mazzoldi, A. Andreoni, C., Bianchi, M., Cavallini, A., Laurino, M., Ricotti, L., Iuliano, R., Matteoli, B., Ceccherini-Nelli, L. (2008). Modeling and control of HIV dynamics. Computer Method and Programs in Biomedicine, 89 (2), 162-168. 